Issued by Sandia National Laboratories, operated for the United States Department of Energy by Sandia Corporation.

NOTICE: This report was prepared as an account of work sponsored by an agency of the United States Government. Neither the United States Government, nor any agency thereof, nor any of their employees, nor any of their contractors, subcontractors, or their employees, make any warranty, express or implied, or assume any legal liability or responsibility for the accuracy, completeness, or usefulness of any information, apparatus, product, or process disclosed, or represent that its use would not infringe privately owned rights. Reference herein to any specific commercial product, process, or service by trade name, trademark, manufacturer, or otherwise, does not necessarily constitute or imply its endorsement, recommendation, or favoring by the United States Government, any agency thereof, or any of their contractors or subcontractors. The views and opinions expressed herein do not necessarily state or reflect those of the United States Government, any agency thereof, or any of their contractors.

Printed in the United States of America. This report has been reproduced directly from the best available copy.

Available to $\mathrm{DOE}$ and $\mathrm{DOE}$ contractors from

U.S. Department of Energy

Office of Scientific and Technical Information

P.O. Box 62

Oak Ridge, TN 37831

Telephone: (865)576-8401

Facsimile: (865)576-5728

E-Mail: reports@adonis.osti.gov

Online ordering: http://www.doe.gov/bridge

Available to the public from

U.S. Department of Commerce

National Technical Information Service

5285 Port Royal Rd

Springfield, VA 22161

Telephone: (800)553-6847

Facsimile: (703)605-6900

E-Mail: orders@ntis.fedworld.gov

Online order: http://www.ntis.gov/ordering.htm

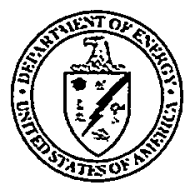




\section{DISCLAIMER}

Portions of this document may be illegible in electronic image products. Images are produced from the best available original document. 
SAND99-1400

Unlimited Release

Printed February 2000

\title{
Robust Bearing Estimation for 3-Component Stations
}

\author{
John P. Claassen \\ Monitoring Technologies Department \\ Sandia National Laboratories \\ P. O. Box 8500 \\ Albuquerque, NM 87185-0655 \\ Fax: (505) 844-5321Ｅ-mail: jpclaas@sandia.gov
}

\begin{abstract}
A robust bearing estimation process for 3-component stations has been developed and explored. The method, called SEEC for Search, Estimate, Evaluate and Correct, intelligently exploits the inherent information in the arrival at every step of the process to achieve near-optimal results. In particular, the approach uses a consistent framework to define the optimal time-frequency windows on which to make estimates, to make the bearing estimates themselves, to construct metrics helpful in choosing the better estimates or admitting that the bearing is immeasurable, and finally to apply bias corrections when calibration information is available to yield a single final estimate. The algorithm was applied to a small but challenging set of events in a seismically active region. It demonstrated remarkable utility by providing better estimates and insights than previously available. Various monitoring implications are noted from these findings.
\end{abstract}

Key words: bearings, estimation, polarization, corrections, location, association

This report in manuscript form has been submitted to the Journal of Pure and Applied Geophysics for publication. 
Intentionally Left blank 


\section{Acknowledgments}

The author is grateful to Timothy McDonald, Mark Harris, Eric Chael, and Greg Elbring of Sandia National Laboratories for providing helpful reviews of this work. The waveforms and event truth used in this work were contributed by D. B. Carr of Sandia National Laboratories. Special thanks go to Mark Harris and Patrick Vaughan for assisting in the preparation of the graphics. Sandia is a multiprogram laboratory operated by Sandia Corporation, a Lockheed Martin Company, for the United States Department of Energy under Contract DE-AC-94AL85000. 
Intentionally Left blank 


\section{Table of Content}

No. Title

Page

1.0

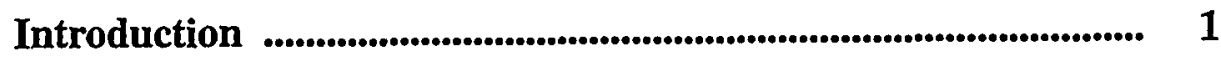

$2.0 \quad$ The Basis for Implementing the SEEC Method ............................. 2

2.1 The Design of the Quadrature Filter Bank ..................................... ' 2

2.2 Searching for the Polarized Regions in the Waveforms ............... 4

2.3 Estimating the Bearings in the Same Mathematical Framework 7

2.4 Evaluating and Correcting the Estimates ........................................ 9

3.0 Application of SEEC to Event Data ................................................. 10

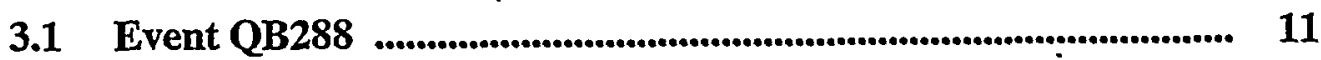

3.2 Event EQ246 .............................................................................................. 11

3.3 Event EQ031 ........................................................................................ 12

3.4 Events EQ133 and EQ134 .............................................................. 12

3.5 Event QB151 ........................................................................................ 13

3.6 The Advantages in Correcting for Bias over Frequency ........... 13

4.0 Discussion of the Approach and the Results ....................... 13

5.0 Conclusions and Monitoring Implications ...................................... 15

$6.0 \quad$ References .......................................................................................... 16

7.0 Tables Captions............................................................................................ 19

$8.0 \quad$ Figure Captions ................................................................................... 20

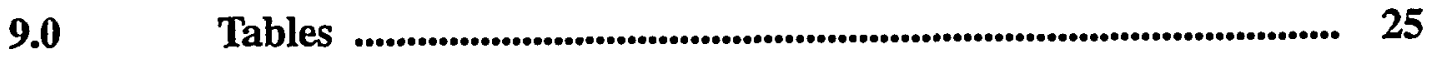

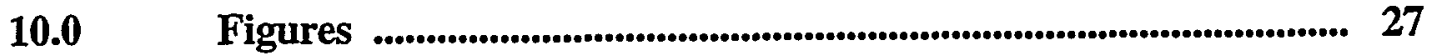


Left Intentionally Blank 


\subsection{Introduction}

As the monitoring thresholds of global and regional networks are lowered, bearing estimates become more important to the processes which associate sparse detections and which locate events. Current methods of estimating bearings from observations by 3-component (3-c) stations lack both accuracy and precision [Alewine, 1992][Koch \& Kradolfer, 1997]. Methods are required which will develop all the precision inherently available in the arrival, determine the measurability of the arrival, provide better estimates of the bias induced by the medium, permit estimates at lower SNRs, provide an indication of the precision of the estimate, and develop insights into the effects of the medium on the estimates.

Various approaches have been advocated to estimate the polarization properties and bearings associated with seismic arrivals. The theoretical basis for defining the polarization properties of signals appears to have arisen from the seminal work of Born and Wolf [1975] where analytical (complexvalued) signals and notions of coherency were introduced for narrowband signals. It was perhaps this work that motivated Samson [1983] to define the degree to which signals are polarized in higher dimensional spaces. Later efforts by Vidale [1986] reminded us how important it is to define polarization in terms of analytical signals and that the general vibrational modes associated with three component observations are elliptically polarized. Magotra, et. al. [1987] showed how the horizontal components of an entire wavetrain could be used to estimate back-azimuths. Here the polarity of the $\mathrm{z}$ component relative to the horizontal signal components was used to remove the azimuthal ambiguity. Jurkevics [1988], limiting the analysis of $P$ arrivals to real-valued (as opposed to complex-valued) signals, demonstrated how to construct an observational covariance matrix by averaging the covariance matrix over 3-c elements of an array to realize bearing estimates having more precision. Although Jurkevics advocated the use of polarization properties to isolate intervals on which to make bearing estimates, no viable rationale to isolate the appropriate estimation interval was offered. Indeed, Jurkevics suggested using signal frequency bands having the best SNR relative to the background noise as a method for defining a measurement interval. However, this approach fails in practice because it does not account for signal-induced noise. In a more recent work Walck and Chael [1991] evaluated various combinations of frequency bands and time-windows in the context of four bearing estimation techniques [Magotra, 1987][Flinn, 1965][Christoffersson, et. al., 1988][Roberts, et. al., 1989] to demonstrate the bearing estimation capability of the three component RSTN. They showed that bearings for events in Eastern North America could be estimated with an accuracy of 6 degrees rms if the estimates from station RSSD, residing on a "pathological" geology, are eliminated. In a notable work by Lilly and Park [1995] complex-valued orthogonal Slepian wavelets were used to elegantly estimate the polarization properties of seismic signals.

The 3-c bearing estimation method disclosed in this work differs from prior efforts in that it makes the following assumptions: 1 ) not all frequencies or time intervals will be equally effective in producing measurable bearings, 2) only those time-frequency windows having good polarization properties will result in precise estimates, 3) the estimates themselves may be frequency 
dependent, 4) those estimates having the largest effective degrees of freedom (a confidence measure) will experience the greatest variance reduction and are the best candidates to combine for a refined estimate which may include bias corrections, and 5) the bearing estimates associated with an event from a given epicentral region will, in general, require correction for frequency dependent lateral refraction or diffraction. A bearing estimation method, called SEEC for Search, Estimate, Evaluate, and Correct, was developed on these underlying assumptions. Earlier disclosures of this technique may be found in Claassen [1998a] [1998b].

In the following section the SEEC methodology is disclosed. To evaluate the bearing estimator the method was applied to a small but challenging set of regional events observed in an active tectonic region of the western United States by a station located near Pinedale, Wyoming. In subsequent sections the results of this evaluation are described and discussed. To assist in the interpretation of the results, various geological features in and about the station are described. In a final section conclusions regarding the effectiveness of the SEEC method are offered and several monitoring implications are highlighted.

\subsection{The Basis for Implementing the SEEC Method}

The block diagram of Figure 1 provides an overview of the SEEC approach. Three-component signals are spectrally decomposed by a quadrature filter bank. The resulting quadrature time series are then searched over short time intervals for suitable polarization properties. SEEC makes bearing estimates on the favorable time intervals of adequate duration and also constructs metrics helpful in evaluating and selecting estimates or discarding all estimates when none is possible. Bias corrections are applied to the accepted estimates and a single final estimate is then constructed from the bias-corrected estimates in a near-optimal way.

This section details the basis for implementing the SEEC method. It addresses the procedure by which to design the quadrature filter bank. Several useful polarization measures are introduced. A pseudo SNR estimator is constructed from these measures. The manner in which the SNR estimator is used to search the 3-c records for the more favorably polarized regions in time and frequency is described. Two related methods of estimating the bearings on contiguous well-polarized timefrequency intervals and their relative utility are then presented. Finally, a method of evaluating and selecting the estimates having better precision and a means of correcting them for the bias introduced by the earth are addressed.

\subsection{The Design of the Quadrature Filter Bank}

To search spectral domains for those time intervals having the best polarization properties, the 3-c signals were filtered by a quadrature digital filter bank whose individual filters are based on orthogonal Slepian wavelets [Lilly \& Park, 1995] having a constant time-bandwidth product (TB). The wavelets were weighted (tapered) with a Hanning window to reduce spectral leakage into the filter 
sidebands. Experience with actual signals had demonstrated that the reduction of leakage from adjacent sidebands is essential to preventing signals in adjacent bands from influencing the bearing estimate in the band of interest. The constant TB property of the filters permits one to logarithmically space the filter center frequencies for efficient spectral coverage. The rationale for defining the individual filters in the bank using the notation of Lilly and Park [1995] is described below.

If the entire frequency interval of interest is defined by $\left[f_{1}, f_{2}\right]$ and $N$ is the desired number of filters to cover that bandwidth, the frequency separation for the filters in logarithmic units can be specified by

$$
d_{f}=\log \left(\frac{f_{2}}{f_{1}}\right) /(N-I)
$$

The filter center frequencies are, therefore, given by

$$
f_{c}(n)=10^{\log \left[f_{1}+(n-1) d_{f}\right]}
$$

where $n=1,2, \ldots, N$. If $p$ is defined as the target $T B / 2$ product and $p_{o}$ is defined as the target center frequency-time product for the filter, then the required half bandwidth for the $n$th filter is

$$
f_{w}(n)=p f_{c}(n) / p_{o}
$$

and the required filter length in samples is

$$
M_{w}(n)=\operatorname{nint}\left[p f_{s} / f_{w}(n)\right]
$$

where nint is the nearest integer function and $f_{s}$ is the sampling frequency. If $M_{w}(n)$ is even, its order is increased by 1 to simplify the alignment of the signals emerging from digital filters having different lengths. The actual resulting wavelet parameters are given by

$$
p_{c}(n)=f_{c}(n) M_{w}(n) / f_{s}
$$

and

$$
p_{w}(n)=f_{w}(n) M_{w}(n) / f_{s}
$$

$p_{c}(n)$ and $p_{w}(n)$ will closely approximate $p$ and $p_{o}$, respectively, for every $n$, but will be constrained by the integral property of $M_{w}(n)$. 
As shown by Lilly and Park, the parameters $M_{w}(n), p_{c}(n)$, and $p_{w}(n)$ are used to define a Toeplitz matrix whose eigenvectors define a family of orthogonal wavelets centered at $f_{c}(n)$. Adjacent pairs of eigenvectors form quadrature filters having almost identical bandpass characteristics. Although the dominant eigenvector is the solution to the posed bandwidth maximization problem, the subsequent eigenvector pairs can be used to expand the frequency band to the target width. In the filtering operation, quadrature filter pairs contribute statistically orthogonal complex-valued (analytic) signals centered on $f_{c}(n)$. The quadrature signals after filtering with tapered filters become statistically independent and have a slightly larger bandwidth.

A provision was included in SEEC to partially whiten the signals with a low order prediction method thereby reducing leakage of dominant spectral content into the desired passband when a strong narrowband signal is processed. This technique was found to be essential in extracting good estimates at the lowest frequencies, which are sensitive to estimation error resulting from leakage of unwanted frequencies as well as from intense background noise.

Setting the filter parameters to $p=5$ and $p_{o}=2$ appeared to work best for signals sampled at 50 $\mathrm{Hz}$. Although these parameters should produce 3 quadrature pairs for every center frequency to realize the specified bandwidth (See Lilly and Park, 1995), the quadrature pairs were limited to two. This choice, after Hanning weighting, not only provided the proper frequency overlap between filter bands but also provided suitable spectral resolution to define a bearing unique to that center frequency (Recall the spectral leakage problem). The band of interest ranged from $0.5 \mathrm{~Hz}$ to $15 \mathrm{~Hz}$ and was covered by 12 sets of quadrature filters. The spectral characteristic of this filter bank is shown in Figure 2. The Hanning taper reduced filter sidelobes to about $-50 \mathrm{~dB}$ relative to the central passband compared to the $-25 \mathrm{~dB}$ that occurred without tapering.

\subsection{Searching for the Polarized Regions in the Waveforms}

The frequency band centered on frequency $f_{c}$ having half bandwidth $f_{w}$ is spanned by $M>0$ quadrature filter pairs ( $M$ is not related to $M_{w}$ ). The argument $n$ is dropped at this point for convenience. Define a vector of $2 k+1$ sequential quadrature (complex-valued) signal samples centered on the sample $j$ from the $i$ th sensor axis and $m$ th quadrature filter as

$$
s_{i m}\left(f_{c^{\prime}} j\right)=\left[s_{i m}(j-k) s_{i m}(j-k+I) \ldots s_{i m}(j) \ldots s_{i m}(j+k-1) s_{i m}(j+k)\right]^{T}
$$

where $i \varepsilon\{x, y, z\}, \mathrm{m}=1,2, \ldots, \mathrm{M}$, and $T$ is the transpose operator. Here bold symbols will denote vectors and matrices. Vectors of samples from the $M$ quadrature filters are used to prepare a rectangular observation matrix of the form 


$$
R\left(f_{c^{\prime}}, j\right)=\left[\begin{array}{ccc}
s_{x I}\left(f_{c^{\prime}}, j\right) s_{y I}\left(f_{c^{\prime}}, j\right) & s_{z I}\left(f_{c}, j\right) \\
\cdot & \cdot & \cdot \\
\cdot & \cdot & \cdot \\
\cdot & \cdot & \cdot \\
s_{x M}\left(f_{c}, j\right) s_{y M}\left(f_{c}, j\right) s_{z M}\left(f_{c}, j\right)
\end{array}\right]
$$

Each vector row contributes additional degrees of freedom to the observation matrix through additional frequency content. The singular values $\lambda_{l}$ and right singular vectors $e_{l}$ of $R$, where $l=1,2$, or 3 , are used to form polarization measures which are used to search intervals of time having suitable polarization properties. The measures found to be useful in defining polarized signal segments are defined below.

These include the degree of polarization

$$
D O P\left(f_{c}, j\right)=\frac{\frac{1}{\sqrt{2}} \sqrt{\left(\lambda_{1}-\lambda_{2}\right)^{2}+\left(\lambda_{2}-\lambda_{3}\right)^{2}+\left(\lambda_{3}-\lambda_{1}\right)^{2}}}{\lambda_{1}+\lambda_{2}+\lambda_{3}}
$$

the degree of linear polarization

$$
\operatorname{DoL}\left(f_{c}, j\right)=\frac{\operatorname{Real}^{2}\left(e_{1 x}\right)+\operatorname{Real}^{2}\left(e_{1 y}\right)+\operatorname{Real}^{2}\left(e_{1 z}\right)}{\left\|e_{1}\right\|^{2}} .
$$

where $e_{1}$ is the dominant singular vector, and the degree of dyadicy

$$
D O D\left(f_{c}, j\right)=\frac{\lambda_{1}}{\lambda_{1}+\lambda_{2}+\lambda_{3}}
$$

The definition of the degree of linear polarization $(D O L)$ is thought to be unique to this work. It is premised on the fact that linear polarization must be defined by real-valued singular vectors. The DOL polarization measures how close the dominant eigenvector is to being real valued. This definition of "linearity" is important to characterizing a propagation environment in which multipath, mode conversion, and scattering introduce noise which may be correlated with the linearly polarized transmission.

From the above definitions various pseudo signal to noise ratios (SNR) can be constructed to include 


$$
S N R_{I}\left(f_{c}, j\right)=\frac{D O D\left(f_{c}, j\right)}{1-D O D\left(f_{c}, j\right)}
$$

and by analogy

$$
\operatorname{SNR}_{2}\left(f_{c^{\prime}}, j\right)=\frac{\operatorname{DOP}\left(f_{c^{,}} j\right)}{1-\operatorname{DOP}\left(f_{c}, j\right)}
$$

These definitions of SNR actually account for noise components which may be uncorrelated or correlated with the signal. The pseudo SNRs are based on coherency-like notions but are not strictly rigorous. This is readily understood from Equation 12 for the case of non-coherent noise and a stationary polarized signal. Under these assumptions, $\lambda_{1}=\sigma_{s}^{2}+\sigma_{n}^{2}$ and $\lambda_{2}=\lambda_{3}=\sigma_{n}^{2}$ where $\sigma_{s}^{2}$ is the signal variance and $\sigma_{n}^{2}$ is the noise variance. In this case $S N R_{l}$ becomes

$$
S N R_{l}\left(f_{c}, j\right)=\frac{\sigma_{s}^{2}+\sigma_{n}^{2}}{2 \sigma_{n}^{2}}
$$

When the SNR becomes large, this pseudo SNR approaches half the actual SNR.

When estimating the polarization properties the lengths of the signal vectors contributing to $R$ were restricted to a fraction of the filter length, typically $1 / 4$ or $1 / 2$. At each center frequency this selection assures that the polarization properties of the signal can be identified with sufficient temporal resolution while retaining one or two cycle(s) of the filtered waveform to fill $\boldsymbol{R}$ (see Equation 8).

To illustrate the screening capability of two of the above polarization measures, the polarization indicators, $D O P$ and $D O L$, were computed and plotted for a regional $\mathrm{P}$ arrival. These properties were portrayed by gray-tone encoding the intensity of the measure in a two dimensional setting where time runs horizontally and frequency vertically. In interpreting these polarization spectrograms, the reader is cautioned that a polarization response can occur before the actual onset of the signal since the quadrature filters are non-causal.

The intensity plot of Figure 3 illustrates the degree to which polarization $(D O P)$ is localized in time and frequency for a $\mathrm{P}$ arrival. Since the actual type of polarization is not indicated by this measure, $D O P$ must be assisted by the test for linear polarization. The plot of Figure 4 indicates that the $D O L$ delineates the linearly polarized regions clearly. It is not surprising to see that both the background noise and signal frequently exhibit linear polarization content over short time intervals. However, the waveform energy must be concentrated in the principal component if it is to be truly linearly polarized. The $D O D$ is capable of demonstrating this concentration. Since an empirical investigation indicated that the $D O P$ is highly correlated with $D O D, D O P$ may be as a substituted for $D O D$. 
It was also observed that the $D O P$ resolves polarization in time and frequency better than does the DOD.

In view of the properties noted above, the interval selection process was actually based on a pseudo SNR defined in the following way

$$
S N R_{3}\left(f_{c^{\prime}}, j\right)=\frac{\sqrt{D O P\left(f_{c^{\prime}}, j\right) D O D\left(f_{c^{, j}}\right)} D O L_{x y}\left(f_{c^{,}} j\right)}{1.0-\sqrt{D O P\left(f_{c^{\prime}} j\right) D O D\left(f_{c^{\prime}} j\right)} D O L_{x y}\left(f_{c^{\prime}} j\right)}
$$

The use of both $D O P$ and $D O D$ through a geometric mean assures that both measures influence the interval selection process with $D O P$ tending to increase the resolution. In addition $D O L$ was reformulated as

$$
D O L_{x y}\left(f_{c}, j\right)=\frac{\operatorname{Real}^{2}\left(e_{1 x}\right)+\operatorname{Real}^{2}\left(e_{1 y}\right)}{\left|e_{1 x}\right|^{2}+\left|e_{1 y}\right|^{2}}
$$

This form of $D O L$ was motivated by the fact that the SVD algorithm conveniently returned a realvalued $z$ component for the principal eigenvector (The $z$-component served as the phase reference for the eigenvector). Hence, this form for $D O L$ slightly heightens the sensitivity of $\mathrm{SNR}_{3}$ to linear polarized components by imposing real valuedness on the two horizontal components which really convey the bearing information.

\subsection{Estimating the Bearings within the Same Mathematical Framework}

Bearing estimates can be formed in a number of ways as discussed by Walck and Chael [1991]. In this work the estimates of the bearing and apparent incident angles are based on the right principal singular vector of a rectangular observation matrix (Equation 8) associated with a selected window simply because the method is compatible with the formulation for the polarization search parameters. Contiguous points are regarded as candidates for a bearing estimate if the pseudo $\operatorname{SNR}_{3}\left(f_{c}, j\right)$, now denoted as $r\left(f_{c}, j\right)$, exceeds one for every $j$ and the duration of the concatenated sub-intervals is longer than two cycles. Suppose the points $j \varepsilon\{J-L, J-L+1, \ldots, J, \ldots, J+L-1, J+L\}$ satisfy this SNR criteria. On such an interval, two approaches, both based on the principal component method, were taken to estimate the angles.

The first approach makes an estimate on the sub-interval of $2 k+1$ points for each $j$. Let the collection of resulting bearing and incident angle estimates be given by $[\phi(j), \theta(j)], j \varepsilon\{J-L, J-L+1, \ldots, J$, ..., $J+L-1, J+L\}$. To assure physically realizable incident angles, when $\theta(j)<90^{\circ}$ for any $j$, set $\theta(j)$ $=180^{\circ}-\theta(j)$ and $\phi(j)=\phi(j)+180^{\circ}$. This action is mathematically justified by the fact that if $\boldsymbol{e}$ is 
a singular vector, then $-\boldsymbol{e}$ will also be an eigenvector. Since the pseudo SNR will vary throughout the interval, it is appropriate to estimate the bearing on the basis of a weighted mean given by

$$
\bar{\Phi}\left(f_{c}, J\right)=\left(\sum_{J-L}^{J+L} r\left(f_{c}, j\right) \phi(j)\right) /\left(\sum_{J-L}^{J+L} r\left(f_{c}, j\right)\right)
$$

A variance given by

$$
\operatorname{var}\left[\bar{\Phi}\left(f_{c}, J\right)\right]=\left(\sum_{J-L}^{J+L} r\left(f_{c}, j\right) \phi(j) \phi(j)\right)\left(\sum_{J-L}^{J+L} r\left(f_{c}, j\right)\right)-\bar{\Phi}\left(f_{c}, J\right)^{2}
$$

and an average pseudo SNR given by

$$
\bar{\Phi}=\left(\sum_{J-L}^{J+L} r\left(f_{c^{\prime}}, j\right)\right) /(2 L+1)
$$

may also be associated with $\bar{\Phi}$. Admittedly the estimates of the means and variances are biased because the estimates are based on correlated (overlapping) samples. However, if the variance is small, the mean established in this manner has been found to be acceptable in practice. Furthermore, if the variance is large, the estimate should be discarded anyway.

The second method of estimating the bearing simply constructs an observation matrix over the entire interval centered on $J$ and uses its right principal singular vector and eigenvalues to construct a single estimate $(\Phi, \Theta)$ and its pseudo SNR given by Equation 15 with $j=J$, here denoted as $r_{\Phi}$. The underlying polarization parameters are dictated by the entire interval as opposed to the subintervals. The observation matrix, however, is modified in a special way to emphasize the individual rows having a better pseudo SNR, namely,

$$
R\left(f_{c^{\prime}} f_{w}\right)=W\left[\begin{array}{ccc}
s_{x I}\left(f_{c^{\prime}}(J-L)\right) & s_{y I}\left(f_{c^{\prime}} J-L\right) & s_{z I}\left(f_{c^{\prime}} J-L\right) \\
s_{x M}\left(f_{c^{\prime}} J-L\right) & s_{y M}\left(f_{c^{\prime}} J-L\right) & s_{z M}\left(f_{c^{\prime}}, J-L\right) \\
\cdot & \cdot & \cdot \\
\cdot & \cdot & \cdot \\
\cdot & \cdot & \cdot \\
s_{x I}\left(f_{c^{\prime}}, J+L\right) & s_{y I}\left(f_{c^{\prime}} J+L\right) & s_{z I}\left(f_{c^{\prime}}, J+L\right) \\
\cdot & \cdot & \cdot \\
s_{x M}\left(f_{c^{\prime}} J+L\right) & s_{y M}\left(f_{c^{\prime}} J+L\right) & s_{z M}\left(f_{c^{\prime}}, J+L\right)
\end{array}\right]
$$


where $W$ is a $(2 L+1) M$ by $(2 L+1) M$ weighting matrix given by

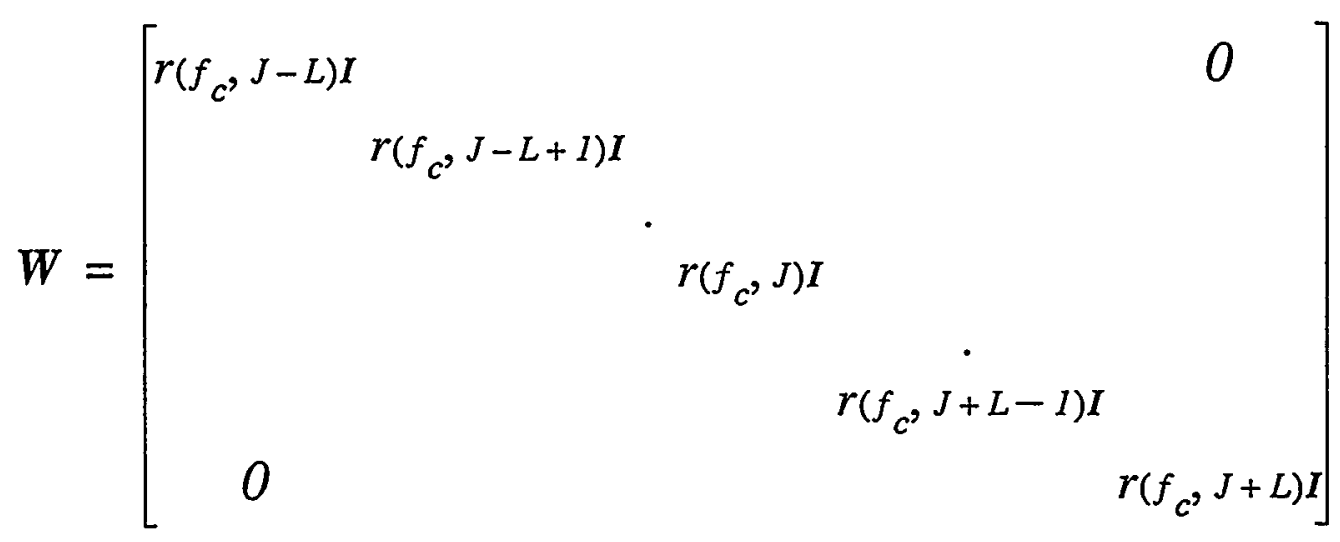

and $I$ is a $M$ by $M$ identity matrix and the individual $r\left(f_{c}, j\right)$ are the pseudo SNRs associated with the individual $2 k+1$ long sub-intervals surrounding each $j \varepsilon\{J-L, J-L+1, \ldots, J, \ldots, J+L-1, J+L\}$. Here, the row elements in Equation 20 are not column vectors.

In practice, estimates of either type having shallow apparent elevation (incident) angles were discarded operationally because the bearings were typically absurd. The use and choice of the two bearing estimators are treated in the following sub-section.

\subsection{Evaluating and Correcting the Estimates}

SEEC yields a bearing for each time-frequency window considered measurable by the criterion described above. To select the better estimates from the entire set of bearings, an information theoretic criterion commonly associated with estimation theory is applied to each estimate. The criterion simply states that the variance of an estimate is reduced by $1.0 /(S N R * T B)$ where $S N R$ and $T B$ characterizes the signals contributing to the estimate. For an example of this principle, see Equation 11 in an article by Shumway and $\mathrm{Kim}$ [1998]. As a consequence, estimates whose $S N R * T B$ exceeds a given threshold are accepted as precision estimates. The others are discarded. In this work $S N R * T B$ is defined as the effective degrees of freedom $(D O F)$ associated with an estimate. Experience with events from the same locale has shown the estimates have utility when $D O F>10$.

Since two estimates are potentially available for each interval satisfying the DOF criterion, a method was constructed to select between the two estimates. Since the standard deviation is a measure of consistency of the bearings (singular vectors) throughout the interval, estimates of either form are only accepted if $\operatorname{var}^{1 / 2}\left[\bar{\Phi}\left(f_{c}, J\right)\right]<15$ degrees. Furthermore, $\bar{\Phi}$ was accepted over $\Phi$ only if $r_{\Phi} T B>10$ and $r_{\bar{\Phi}} T B$ exceeded $r_{\Phi} T B$ by 5 . This choice indicates a strong preference for estimates over the entire interval as opposed the average estimate over many sub-intervals. This presumes that better estimates are possible if the rectangular observation matrix has a large number of effective degrees of freedom. Of course the polarization coherency must be sustained throughout 
the entire interval to build an estimate on self-consistent information. $\operatorname{var}\left[\bar{\Phi}\left(f_{c}, J\right)\right]$ is an indicator as to whether this consistency really exists. This selection process is applied to all time-frequency intervals satisfying the DOF requirement and may result in several estimates or possibly none.

When multiple estimates satisfying the acceptance criterion are available for an event, the effects of frequency dependent bearing biases may have to be addressed. Only then can all the inherent accuracy and precision associated with the accepted bearings be achieved. The biases for an epicentral region of the earth may be determined by using well-located reference events within that region. Suppose that the frequency dependent estimates $\hat{\Phi}_{i}\left(f_{c}\right)$ and their degrees of freedom $D O F_{i}\left(f_{c}\right)$ as observed by a particular station are available for reference events $i=\{1,2, \ldots, I\}$ within the epicentral region. The frequency dependent biases are compared with the true (great-circle) bearings $\Phi_{i}$ to establish the bearing bias in the following near-optimal manner

$$
\operatorname{bias}\left(f_{c}\right)=\frac{\sum_{i}\left[\hat{\Phi}_{i}\left(f_{c}\right)-\Phi_{i}\right] \cdot D O F_{i}\left(f_{c}\right)}{\sum_{i} D O F_{i}\left(f_{c}\right)}
$$

Once the regional bearing biases are established as a function of frequency for a particular station, estimates $\hat{\Phi}\left(f_{c}\right)$ for a subsequent event with effective degrees of freedom $D O F\left(f_{c}\right)$ arising from the same epicentral region may be corrected for bias in the following near-optimal manner

$$
\hat{\Phi}=\frac{\sum_{f_{c}}\left[\hat{\Phi}\left(f_{c}\right)+\operatorname{bias}\left(f_{c}\right)\right] \cdot \operatorname{DOF}\left(f_{c}\right)}{\sum_{f_{c}} D O F\left(f_{c}\right)}
$$

to achieve a single bearing with all the inherently available accuracy and precision. Conceivably the summation could also extend over different time intervals as well as frequency intervals.

\subsection{Application of SEEC to Event Data}

The polarization-based bearing estimator was applied to a reasonably large set of regional $\mathrm{P}$ arrivals arising from events located in the western US and observed by a high frequency station located near Pinedale, Wyoming. The locations of the events analyzed as part of the entire effort appear in the map of Figure 5. The event truth for the events only appearing in this article is listed in Table 
1. The arrivals were extracted from the S-3 seismometer channels, which had been electronically altered to provide an acceleration output having a nominal 0.5 to $80 \mathrm{~Hz}$ passband which was sampled at $200 \mathrm{~Hz}$. The bearing estimates were restricted to the central frequencies associated with the filter bank $(0.5$ to $15 \mathrm{~Hz})$ after having decimated the data to a $50 \mathrm{~Hz}$ sample rate. Contiguous samples were included in a measurable interval if $r\left(f_{c}, j\right)>1$ for every $j$ in the interval. The locations of the events analyzed as part of the entire effort appear in the map of Figure 5. The event truth for the events addressed in this article appears in Table 1.

The bearing error estimates, as inferred from the NEIC locations, are presented here to illustrate the efficacy of the SEEC approach. The SEEC errors are also compared with the errors associated with a conventional broadband estimator whose solutions were based on the initial arrival and the principal eigenvector of a real valued covariance matrix. Except to illustrate the features of the SEEC estimator, only those estimates having effective $D O F>10$ were accepted and plotted. For most of the error plots, a composite polarization indicator related to $S N R_{3}$ appears above the bearing error plot to make evident the SEEC rationale. The composite indicator is defined by

$$
i_{c}=(D O P \cdot D O D)^{1 / 2} D O L
$$

and nears unity on sub-intervals having favorable polarization properties. The bearing error lines extend over the measurable time-intervals as defined by the SNR criterion. The frequency at which the estimate was made is symbol encoded in accord with a key appearing in the attached legend. The key also provides the effective DOF and the actual bearing estimates (PHI). The following events were selected to illustrate the characteristics of SEEC and the influence of the earth's propagation environment on the bearing estimates.

\subsection{Event QB288}

This arrival was produced by a quarry shot in eastern Wyoming at a range of 3.4 degrees. The broadband signal to noise ratio for this arrival is poor as implied by the trace of Figure 6 a. However, SEEC was able to identify spectral components and time intervals having usable polarized states at the middle and lower frequencies as illustrated in polarization spectrogram (Figure 6a). The bearing error graph of Figure $6 \mathrm{~b}$ illustrates that nearly correct estimates can be produced while the broadband approach based on a real covariance matrix fails. Here acceptable estimates primarily appear at the onset of the arrival.

\subsection{Event EQ246}

The estimator was applied to an arrival emanating from a magnitude 5.8 earthquake in southern Utah at a range of 6.4 degrees. The arrival produced strong, linearly polarized content at frequencies less than $3 \mathrm{~Hz}$ as illustrated in Figure $7 \mathrm{a}$. The higher frequency selectivity of the constant TB 
filters at the lower frequencies enabled SEEC to isolate polarized components at frequencies as low as $0.5 \mathrm{~Hz}$, a frequency at which this event would not have been detected.

An interesting bearing dispersion characteristic emerged for this arrival as indicated by the graph of Figure 7b. The bearings move with frequency to the left of the event as observed at the recording station. The most accurate bearing estimates occurred at the lowest frequencies. Perhaps lateral refraction or diffraction causes bearing errors as large as 24 degrees to the left of the Pinedale station at a frequency of $2.35 \mathrm{~Hz}$. Other events in the vicinity of this one exhibited similar bearing dispersion characteristics. In fact this bearing characteristics was evident for events along the Wasatch range and throughout northern Utah. Comparison of the conventional broadband estimate with the SEEC estimates appears to indicate that the broadband estimate is a frequency-weighted average of the SEEC bearings.

\subsection{Event EQ031}

The graph of Figure 8a illustrates the strength of the SEEC approach in finding useful polarized content in time and frequency. Here linearly polarized content was identified in both the first and second $\mathrm{P}$ arrivals over broad frequency intervals. This arrival was produced by a local earthquake that occurred just $1 / 2$ degree east of the Pinedale station.

As evident in Figure $8 \mathrm{~b}$, frequency dependent bearings can also occur at short ranges. The ground truth suggested a reference bearing in the NE quadrant; whereas, the low frequency estimate suggests that event arose from the SE quadrant. The errors have been referenced to an assumed bearing of 107 degrees because a slight error in location for a nearby event causes large errors in bearing. The arrivals appear to experience bearing dispersion to the left of the station, although one cannot state this with complete confidence because the effective DOF are limited. The second $\mathrm{P}$ arrival, however, appears to confirm this possibility, because bearing estimates for the second arrival almost replicate the estimates for the first arrival. This possibly suggests that these two arrivals experienced similar crustal effects.

\subsection{Events EQ133 and EQ134}

Events EQ133 and EQ134 are two nearly co-located earthquakes in the Hebgen Lake area west of Yellowstone Park. The nearly identical waveforms (except for amplitude) provide additional confidence that these two events are co-located. The bearing estimates for these two events are shown in Figures $9 \mathrm{a}$ and $9 \mathrm{~b}$, respectively. The bearings for the two events cluster to the right of the great circle paths with mean biases of 25.9 and 25.4 degrees, respectively. The bearing estimates for these events suggest that the bearings may be slightly dispersed in frequency to the right as well, although orderliness with frequency is not uniquely evident. Comparisons of the estimates for the two events suggests that the SEEC method can reasonably replicate bearings with a precision somewhere between 1.2 to 1.8 degrees 1.8 even though the DOF are limited. Furthermore, a comparison of the mean unweighted bearings for the two events gives an indication of the potential in- 
crease in precision possible when the individual estimates are combined in a suitable scheme. The conventional broadband estimates, whose error are denoted by the arrow heads, fall among the SEEC estimates.

It is interesting to note that even though the amplitude for the initial arrival for event EQ133 was twice that of EQ134 and the estimation TB products are similar, the available DOF did not rise proportionately with the square of the event amplitude. This illustrates that the arrivals contain noise components which are correlated to the signal amplitude. As a consequence, the SNR remains nearly constant.

\subsection{Event QB151}

The error estimate of Figure $10 \mathrm{~b}$ show that the best bearing may not always be available at the onset of the arrival. This arrival arose from a quarry blast in eastern Wyoming but was overridden by the wavetrain of a preceding event. The polarization spectrogram shown in Figure 10a demonstrates that the searching method can find frequency-time intervals that yield measurable estimates in the presence of interference. It is interesting to note that estimates on the initial arrival, whose errors were comparable to the broadband estimate, were also available from the SEEC process but were discarded on the basis of too few $D O F$.

\subsection{The Advantages in Correcting for Bias over Frequency}

The bearing biases were determined for four earthquakes in southern Utah using the method embodied in Equation 22. The estimated biases were then applied to the same event data to remove the biases in accord with Equation 23. The actual statistics were based on the "leave-one-out method" wherein the corrected estimates for an event used biases computed from the remaining events. The results appear in Table 2. The rms accuracy for this small collection of events is 1.6 degrees. This compares favorably with the a rms error 7.6 degrees associated with the individual estimates before creating a weighted average.

This procedure was repeated for 7 events in central Utah. The results are shown in Table 3. SEEC yielded an accuracy of 1.5 degrees rms for the weighted average technique. The accuracy of the collection of individual estimates was 5.9 degrees rms. These results demonstrate that individual low accuracy estimates at different frequencies for a common event can be corrected for bias and optimally combined to realize improved accuracy. The lack of a dramatic improvement is thought to be attributed to the small sample size and the inaccuracies in the ground truth.

\subsection{Discussion of the Approach and the Results}

In the SEEC method quadrature filters spectrally decompose the signals into overlapping bands. The use of wavelets having a constant $T B$ characteristic to define the filters is attractive because the bandwidth is suitably matched to signal and noise environments where narrower filtering is re- 
quired at the lower frequencies to improve the signal to noise ratio. The retention of the phase sensitivity across multiple channels through the use of quadrature filters is important to isolating the coherent signal components in a multipath and scattering propagation environment. Multiple quadrature filters at a given center frequency effectively widen the bandwidth about the center frequency while providing additional independent rows in the observation matrix to enhance the accuracy of the estimate.

A composite polarization search parameter is effective in identifying time-frequency intervals most likely to produce bearing estimates having good precision. A unique and effective linear polarization measure was included in the search parameter. This approach is thought to avoid the limitations associated with fixed temporal and frequency windows or, for that matter, with accepting the detection window as the defining window, either of which may miss important polarized content.

An information theoretic variance reduction parameter associated with the selected intervals is effective in evaluating and selecting the better bearing estimates from the set of defining intervals. This variance reduction parameter was specifically found to be useful in calibrating sub-regions for bearing biases and for correcting subsequent observations for biases in a near-optimal setting. The bias correction uses the select bearing estimates and their corrections in a weighted average scheme to embed as much of the inherent accuracy and precision available into a single final estimate. The results of Table 2 and 3 show the potential effectiveness of this method.

Bearing biases have been noted in many of the observations at the Pinedale station. Of the biased observations, some reflected a strong orderly bearing dispersion with frequency. Bearing dispersion was evident for events arising throughout Utah and from a local event just east of the station. This frequency dependence may be attributable to a wavelength effect where the longer wavelengths are not as sensitive to thin structures as are the shorter wavelengths. Biases have been typically attributed to dipping receiver structures at some stations, an example of which was documented in a recent work by Bear, Pavlis, and Bokelmann [1999] for the 3-component array at Pinon Flats, California. They assert that the azimuthally varying particle-motion anomalies are frequency dependent with some anomalies larger than 40 degrees. However, systematic trends with frequency are not strongly evident in their results.

The results here suggest that more than a simple dipping structure at the receiver accounts for the observed bearings at the Pinedale station. Possibly source, path and receiver structures are all influencing the observations depending on the epicentral region. Some of the complexity in the observations may be attributed to the complexity of the crustal structure in the vicinity of the Pinedale station. As inferred from the work of Smithson, et al. [1979] the Pinedale station is situated on the southwestern edge of the Wind River thrust fault which dips to the northeast beneath the Wind River Mountains and runs parallel to the mountain range. The Wind River thrust fault overrides the Green River sedimentary basin to the southwest of the mountain range. This thrust fault apparently gave rise to the Pacific Creek anticline which is a faulted gentle fold in the basin in front of the Wind River fault. Synclines and anticlines, to include the Pinedale anticline, also surround the sta- 
tion (see for example, Law and Spencer, [1989]). These folded and faulted zones could give rise to some of the observed bearing characteristic.

Another possible explanation for the bearing biases are the horizontal velocity gradients in the upper mantle of the western US. Estimates of these gradients at a depth of $100 \mathrm{~km}$ are illustrated by the color intensity map of Figure 11 . The color-encoded scale covers velocity changes of $\pm 5 \%$ about $8.23 \mathrm{~km} / \mathrm{sec}$. These velocity gradients undoubtedly extrapolate to the uppermost mantle (45 $-60 \mathrm{~km}$ ) and lower crust and therefore influence regional $P$ arrivals. These gradients may account for refractions as great as 25 degrees as inferred from Snell's law. The bearings associated with the events in Colorado could be influenced by the velocity contrast across the Archean-Proterozoic suture zone along the Wyoming-Colorado border where island arcs are thought to have collided with the North American plate [Vernon, et. al., 1998]. Some evidence for this velocity contrast is evident in the gradient map.

\subsection{Conclusions and Monitoring Implications}

These results have shown that it is essential to search the temporal-frequency windows for regions having suitable polarization properties before attempting a bearing estimate. Limiting bearing estimates to frequencies having the best detection SNR may miss useful bearing information. Furthermore, on some occasions the useful time-interval may not occur at the onset of the $\mathrm{P}$ arrival, as a result of interference or an emerging arrival.

SEEC is premised on the assumption that the estimates may be frequency dependent. The method is therefore capable of measuring bearing dispersion, thereby providing the bearing bias as a function of frequency. In view of the frequency dependence, it is important to avoid contaminating a bearing estimate at a given frequency through leakage into the sidebands of a filter. Filters having low sidelobe characteristics, as a consequence, are essential to realizing unbiased estimates. When quality filtering is inadequate as in the case of strong, narrow band signals, it is helpful to partially whiten the signals of interest before bandpass filtering. For measurable events SEEC can provide additional insight at differing time-frequency windows to make appropriate judgements for constructing a single final estimate in comparison to conventional approaches which directly produce a single outcome without regard to the effects of the underlying frequency content.

In addition, bearings should be estimated on as many time and frequency intervals as possible to realize the inherently available precision. To assist in achieving accuracy, near-optimal methods were identified to measure the frequency dependent biases for a given locale and to correct subsequent estimates from that locale for frequency dependent biases. The near-optimally combined and corrected bearing estimates at various time-frequency intervals were shown to improve the accuracy over that associated with making bias corrections at the individual frequencies. An information theoretic information criterion was found to be helpful in evaluating and selecting the higher precision bearings before making the bias inferences or corrections. 
There must be an awareness that bearing estimates can be frequency dependent in an operational setting. To do otherwise is to accept poor precision in the estimates for events arising from the same locale simply because the estimates will be effected by the differing frequency content from event to event. Further, making bearing corrections without accounting for the frequency content in the estimate may produce inaccurate corrections.

There must also be an awareness that not all arrivals will yield useful bearing estimates. The SEEC approach provides insight to the measurability of an event. When an arrival is measurable, a careful search in frequency and time for polarized content is essential to identifying useful estimation intervals. Estimates at a low frequency, when available, will often yield a near bias-free estimate. The lowest frequency estimate is probably the most useful bearing in associating detections when building events. When locating events, bearings will make a better contributions when the estimates are appropriately selected and have their respective biases removed to form a weighted average of the corrected bearings.

In the future the utility of SEEC method should be further established on larger data sets which include observations in stable as well as tectonic regions. This effort should "tune" the parameters in the algorithm for optimal performance. The resulting algorithm should then be compared with operational bearing estimation methods. If SEEC demonstrates to be an effective approach, methods of integrating the technique in an operational context must also be considered. Other efforts should also consider extending SEEC to other types of polarized arrivals, to codas, and to arrays. It is anticipated that the SEEC algorithm will eventually appear in MatSeis, a publicly available seismic analysis tool [Harris \& Young, 1996].

\subsection{References}

Alewine, R. W. (1992), The role of arrays in global monitoring systems, Proc. of the GERESS Symposium, Waldkirchen, pp. 2224

Bear, L. K., G. L. Pavlis, and G.H. R. Bokelmann, Multi-wavelet analysis of three-component seismic arrays: Application of measure effective anisotropy at Pinon Flats, California, Bull. of the Seismological Soc. of America, 89, 693-705

Born, M. and E. Wolf (1975), Principles of Optics, Pergamon Press

Christoffersson, A., E. S. Husebye, and S. F. Ingate (1988), Wavefield decomposition using MLprobabilities in modeling single-site 3-component records, Geophysics J., 93, 197-213 
Claassen, J. P. (1998a), In search of a robust 3 component bearing estimator, 93rd Annual Meeting of the Seismological Soc. of America., Boulder, CO

Claassen, J. P. (1998b), Robust and intelligent bearing estimation, Proc. of 20th Annual Seismic Research Symposium on the Monitoring a CTBT, Santa Fe, NM, 495-504

Flinn, E. A. (1965), Signal analysis using rectilinearity and direction of particle motion, Proc. IEEE, 53, 1884-1876

Harris, M. and C. Young (1996), MatSeis: A Seismic GUI and Toolbox for MATLAB, Seismol. Res. Lett., 67(2), 267-269

Jurkevics, A. (1988), Polarization analysis of three-component array data, Bull. of the Seismological Soc. of America, 78, 1725-1743

Koch, K., and U. Kradolfer (1997), Investigation of azimuth residuals observed at stations of the GSETT-3 alpha network, Bull. of the Seismological Soc. of America, 87, 1576-1597

Law, B. E. and C. W. Spencer (1989), Geology of tight gas reservoirs in the Pindeale anticline area, Wyoming , U.S. Geological Survey Bulletin 1886

Lilly, J. M and J. Park (1995), Multiwavelet spectral and polarization analysis of seismic records, Geophysical J. International, 122, 1001-1021

Magotra, N., N. Ahmed, and E. P. Chael (1987), Seismic event detection and source location using single station (three component) data, Bull. of the Seismological Soc. of America, 77, 958-971

Roberts, R. G., A. Christoffersson, and F. Cassidy (1989), Real-time event detection, phase identification, and source location estimation using single-station three component seismic data, Geophysics J., 97, 471-480

Samson, J. C. (1983), The spectral matrix, eigenvalues, and principal components in the analysis of multi-channel geophysical data, Annals Geophysicae, 1, 115-119 
Shumway, R. H. and S. E. Kim (1998), Detection and estimation on small infrasonic arrays, Proc. of the 20th Annual Seismic Research Symposium on Monitoring a CTBT, pp 628-637

Smithson, S. B., J. A. Brewer, S. Kaufman, J. E. Oliver, and C. A. Huruch (1979), Structure of the Laramide Wind River Uplift, Wyoming, from the COCORP deep reflection data and from gravity data, J. of Geophysical Research, 84, B11, 5955-5972

Vernon, F., K. Dueker, and G. Pavlis (1998) Shakedown at the Gates of the Lodore, IRIS Newsletter, $17,1-4$

Vidale, J. E. (1986), Complex polarization analysis of particle motion, Bull. of the Seismological Soc. of America, 76, 1393-1405

Walck, M. C. and E. P. Chael (1991), Optimal backazimuth estimation for three-component recordings of regional seismic events, Bull. of the Seismological Soc. of America, 81, 643-666 


\subsection{Table Captions}

Table 1. The ground truth for events appearing within this article

Table 2. The bearing errors after correction for regional biases are shown here for events occurring in southern Utah. The DOF reflects the accumulative precision from estimates contributing at different frequencies and time intervals for a given. event

Table 3. The bearing errors after correction for regional biases are shown here for events occurring in the middle of Utah. The accumulated effective $D O F$ associated with each event is also indicated. 


\section{$\underline{\text { 8.0 Figure Titles }}$}

Figure 1. A block diagram of the SEEC bearing estimation process is shown here. The quadrature filter bank decomposes the 3-component signals into overlapping spectral bands. A search process finds those time-spectral intervals having favorable polarization properties. Bearing estimates are made on those intervals. The estimates having experienced sufficient variance reduction are included in a frequency dependent bias correction process to achieve near-optimal results.

Figure 2. The bandpass characteristics of the filters in the quadrature filter bank are illustrated here. The individual filters, characterized by a constant TB product, have center frequencies which are logarithmically spaced to efficiently cover the band of interest. Frequency decomposition of the incoming signal is important because, as shown in this work, bearings can be strongly frequency dependent.

Figure 3. This gray-scale encoded contour plot illustrates how the degree of polarization $(D O P)$ can localize time and frequency intervals having highly polarized content. These estimates were formed from the complex valued signals produced by the filter bank illustrated in Figure 2. When the DOP approaches one, the signal is regarded as highly polarized. On the other hand when the DOP approaches zero, the signal is regarded as unpolarized. The vertical component of the signal contributing to the DOP estimates appears in the trace above and has been 
synchronized with the $D O P$ estimates. Be aware that a polarization response can occur before the on set of the signal because the filters are non-causal.

Figure 4. This gray-scale encoded contour plot illustrates how the degree of linear polarization $(D O L)$ as defined in this work provides additional information for locating polarized content for the same event illustrated in Figure 3. Whenever DOL approaches one within a time-frequency interval, the signal or noise is potentially linearly polarized. Rigorously, those intervals are only linearly polarized when the degree of dyadicy nears one. This polarization measure together with others serve together in selecting favorable intervals on which to make bearing estimates.

Figure 5. The location of events contributing to an evaluation of the SEEC algorithm are shown above. Earthquakes (EQ) are designated by shaded diamonds and quarry blasts (QB) by asterisks. Not all of the events shown here are addressed within this paper but are located on the map to illustrate that the SEEC algorithm was applied to a reasonably large set of events sampling different paths. The location of the Pinedale station, whose observations contributed to the evaluation, is identified by a shaded square.

Figure 6. a) Appearing in the upper graph is a gray-scaled encoded composite polarization indicator which identifies several disjoint windows in time and frequency having weak linear polarized content for event QB288. The indicator approaches one when linearly polarized content is present. The signal from the vertical seismometer appears above the polarization measure. b) The lower graph illustrates the bearing errors associated with frequency-time intervals carefully defined by the polarization indicator. Error measures are extended over the 
estimation interval and are only illustrated for estimates having effective degrees of freedom $(D O F)$ exceeding 10 . The solid bar denote the bearing error associated with an estimate based on the entire available spectrum as opposed to the frequency intervals defined by the quadrature filter bank. This result demonstrates that the SEEC approach is effective in defining useful intervals on which to make accurate bearing estimates.

Figure 7. a) The composite polarization indicator finds significant polarized content for event EQ246 at frequencies less than $3.0 \mathrm{~Hz}$. The selectivity of the filter bank was effective in isolating polarized components at the lowest frequencies over long time intervals. The corresponding signal from the vertical seismometer appears above the polarization spectrum. b) The lower graph illustrates the bearing errors associated with the estimation intervals having $D O F$ greater than 10 . Bearing dispersion to the left of the great circle path is evident in the error characteristic. The lower frequencies clearly experienced less frequency dependent refraction or diffraction along this Utah to Wyoming path as one would expect.

Figure 8. a) The composite polarization indicator finds linear polarized content within two time intervals for an arrival associated with a local earthquake, EQ031. The corresponding arrivals are evident in the vertical seismometer trace above the polarization spectrum. b) Bearing errors appear in the lower graph. The acceptance threshold $(D O F)$ was lowered to illustrate the bearing errors for the weak second arrival. Both arrivals appear to confirm that the bearing dispersion occurs to the left 
of the presumed line of sight for both the first and second $\mathrm{P}$ arrival. This result illustrates that frequency dependent bearings can also be observed at short ranges and in this case is induced by shallow structures.

Figure 9. a) An earthquake (EQ133) near Hebgen Lake yields a relatively tight but heavily biased bearing error pattern when observed at the Pinedale station. b) A nearly co-located earthquake (EQ134) yields a similar bearing pattern. A comparison of the error estimates for these two events provides a quantitative indication of the relative precision ( -1.0 degrees) with which 3-c estimates can be made with the SEEC process. It is interesting to note that even though the available power in event EQ134 was less than 1/4 than that in event EQ133, the effective degrees of freedoms are comparable for those frequency windows having approximately the same time span. This implies that the noise is correlated with the signal amplitude.

Figure 10 a) As observed in the trace above, this arrival experienced interference by a preceding arrival. Despite the poor SNR, the interval search process found several polarized regions in the coda of the primary arrival for event QB151. b) The strongest polarized interval in the $\mathrm{P}$ coda resulted in a single usable estimate having a sufficient number of DOF. A large error is associated with the broadband estimate at the onset of the event. The arrival time was inferred from the NEIC origin time and the $\mathrm{P}$ wave travel-time characteristic. 
Figure 11. The lateral $P$ velocity gradients at a depth of $100 \mathrm{~km}$ across Western United States are illustrated through a color encoded map. Various tectonic features in relationship to the velocity gradients are also labelled. The percent deviations in velocity are referenced to a mean velocity of $8.23 \mathrm{~km} / \mathrm{sec}$. A simple examination, based on Snell's law, suggests that rays could bend laterally by as much as 25 degrees (The graph was provided by the courtesy of Ken Dueker, University of Colorado) 
Table 1.

\begin{tabular}{|c|c|c|c|c|c|c|c|c|}
\hline ID & Latitude & Longitude & State & Date & Time & Mag & Depth & Range \\
\hline EQ246 & 37.090 & -113.472 & UT & $09 / 02 / 92$ & $10: 26: 20.9$ & 5.8 & 15 & 6.43 \\
\hline EQ355 & 37.567 & -112.322 & UT & $12 / 19 / 91$ & $20: 26: 35.7$ & 3.6 & 7 & 5.62 \\
\hline EQ181a & 37.736 & -113.171 & UT & $06 / 29 / 92$ & $01: 59: 06.0$ & 4.1 & 1.4 & 5.75 \\
\hline EQ181b & 37.733 & -113.173 & UT & $06 / 29 / 91$ & $22: 20: 45.8$ & 3.8 & 3.1 & 5.71 \\
\hline EQ081 & 37.816 & -112.995 & UT & $03 / 22 / 91$ & $14: 59: 59.2$ & 3.2 & 2.0 & 5.61 \\
\hline EQ221 & 38.788 & -111.587 & UT & $08 / 09 / 91$ & $08: 49: 20.0$ & 3.5 & & 4.27 \\
\hline EQ176 & 38.783 & -111.554 & UT & $06 / 24 / 92$ & $07: 31: 20.2$ & 4.4 & & 4.27 \\
\hline EQ193 & 38.322 & -111.123 & UT & $07 / 1192$ & $13: 23: 07.8$ & 4.4 & & 4.27 \\
\hline EQ161 & 39.302 & -111.159 & UT & $06 / 09 / 92$ & $23: 30: 18.6$ & 3.4 & 6.1 & 3.7 \\
\hline EQ074 & 39.352 & -111.171 & UT & $03 / 13 / 91$ & $20: 33: 14.5$ & 3.2 & 9.0 & 3.7 \\
\hline EQ303 & 39.340 & -111.161 & UT & $10 / 29 / 92$ & $06: 57: 43.1$ & 2.9 & 1.5 & 3.6 \\
\hline EQ326 & 39.332 & -111.155 & UT & $11 / 19 / 92$ & $06: 46: 57.5$ & 3.0 & 5.7 & 3.6 \\
\hline QB288 & 44.352 & -105.391 & WY & $10 / 14 / 92$ & $17: 31: 52.8$ & & & 3.4 \\
\hline QB151 & 42.780 & -105.610 & WY & $05 / 31 / 91$ & $21: 44: 53.3$ & & & 2.9 \\
\hline EQ031 & 43.020 & -108.947 & WT & $01 / 31 / 92$ & $12: 01: 47.9$ & 3.4 & $5(g)$ & 0.5 \\
\hline EQ133 & 44.533 & -111.312 & MT & $05 / 13 / 91$ & $14: 13: 05.8$ & 3.4 & $5(g)$ & 2.1 \\
\hline EQ134 & 44.438 & -111.217 & MT & $05 / 14 / 91$ & $01: 49: 43.7$ & 3.1 & $5(g)$ & 2.1 \\
\hline
\end{tabular}


Table 2.

\begin{tabular}{|c|c|c|c|c|}
\hline Event & DOF & $\begin{array}{c}\text { Actual } \\
\text { (degrees) }\end{array}$ & $\begin{array}{c}\text { Estimate } \\
\text { (degrees) }\end{array}$ & $\begin{array}{c}\text { Error } \\
\text { (degrees) }\end{array}$ \\
\hline EQ246 & 175.51 & -150.80 & -149.01 & 1.79 \\
\hline EQ081 & 74.78 & -150.90 & -151.21 & --0.31 \\
\hline EQ181a & 43.45 & -149.80 & -148.51 & 1.29 \\
\hline EQ181b & 130.75 & -150.00 & -152.19 & -2.19 \\
\hline
\end{tabular}

Table 3.

\begin{tabular}{|c|c|c|c|c|}
\hline Event & DOF & $\begin{array}{c}\text { Actual } \\
\text { (degrees) }\end{array}$ & $\begin{array}{c}\text { Estimate } \\
\text { (degrees) }\end{array}$ & $\begin{array}{c}\text { Error } \\
\text { (degrees) }\end{array}$ \\
\hline EQ176 & 243.29 & -158.52 & -159.89 & -1.37 \\
\hline EQ221 & 65.34 & -158.17 & -155.52 & 2.65 \\
\hline EQ074 & 105.65 & -159.82 & -158.09 & 1.73 \\
\hline EQ161 & 184.34 & -160.21 & -162.01 & -1.80 \\
\hline EQ303 & 53.65 & -160.00 & -159.40 & 0.60 \\
\hline EQ193 & 187.41 & -160.53 & -160.62 & -0.09 \\
\hline EQ326 & 150.87 & -160.10 & -160.40 & -0.30 \\
\hline
\end{tabular}




\subsection{Figures}

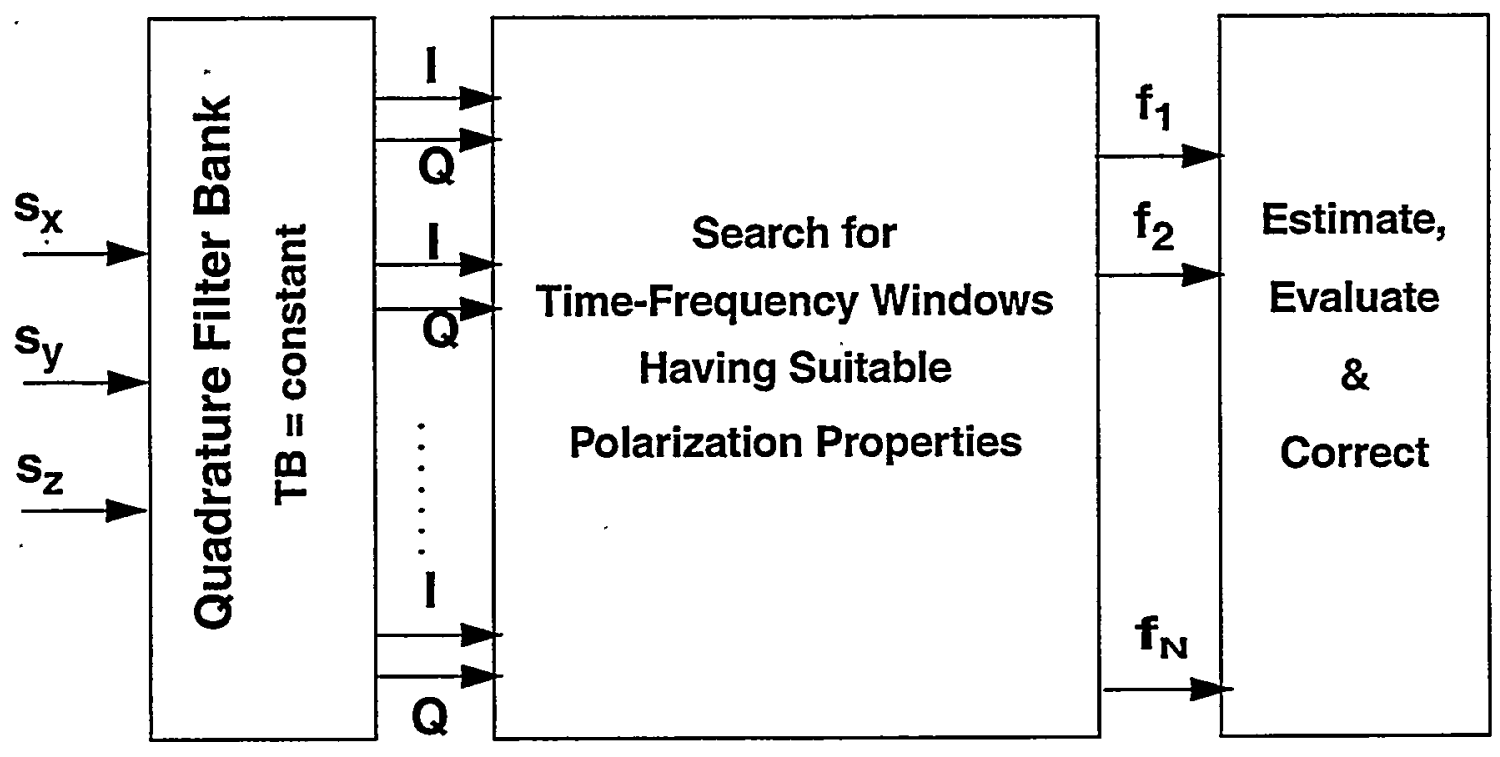

Figure 1. 


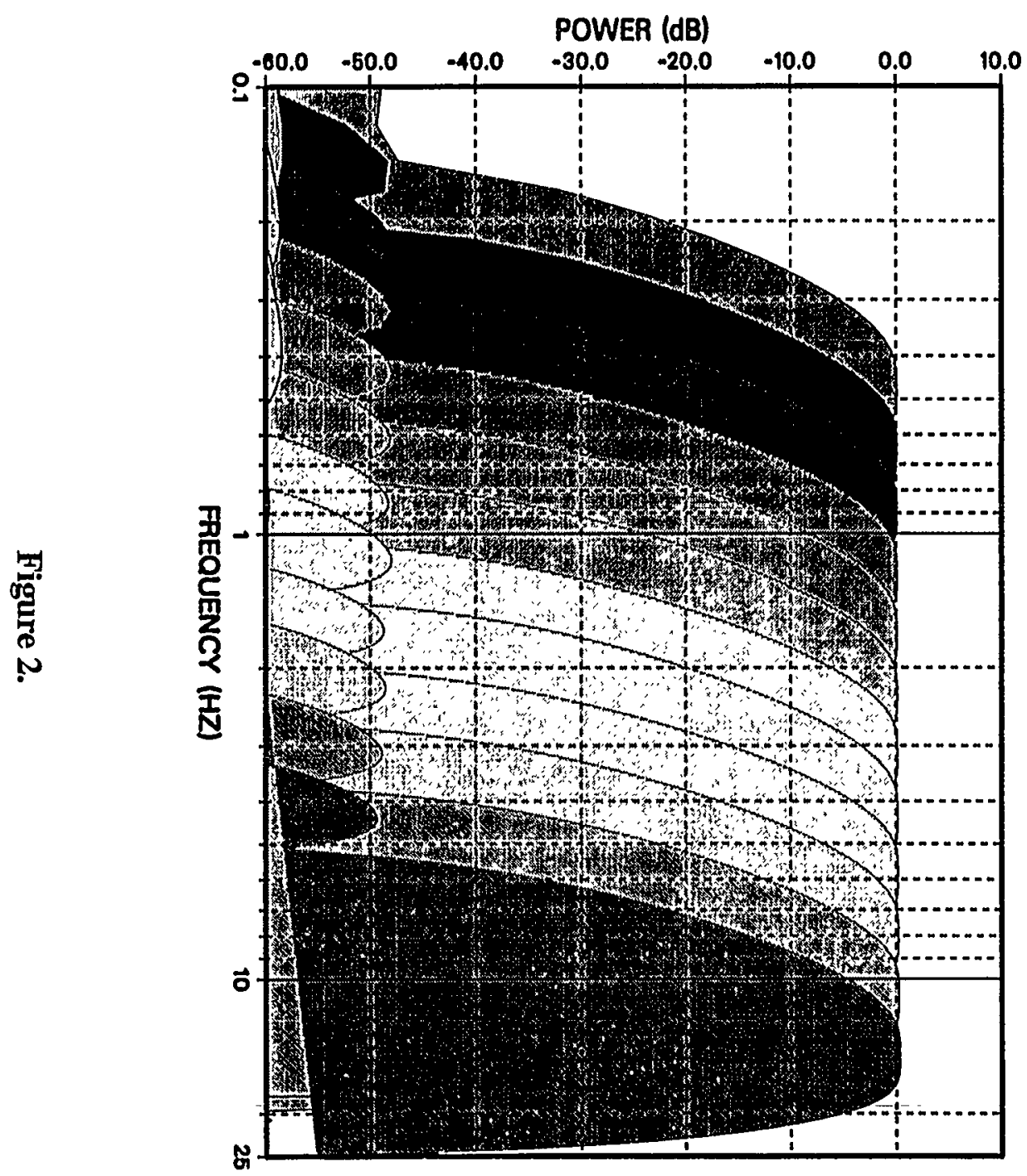




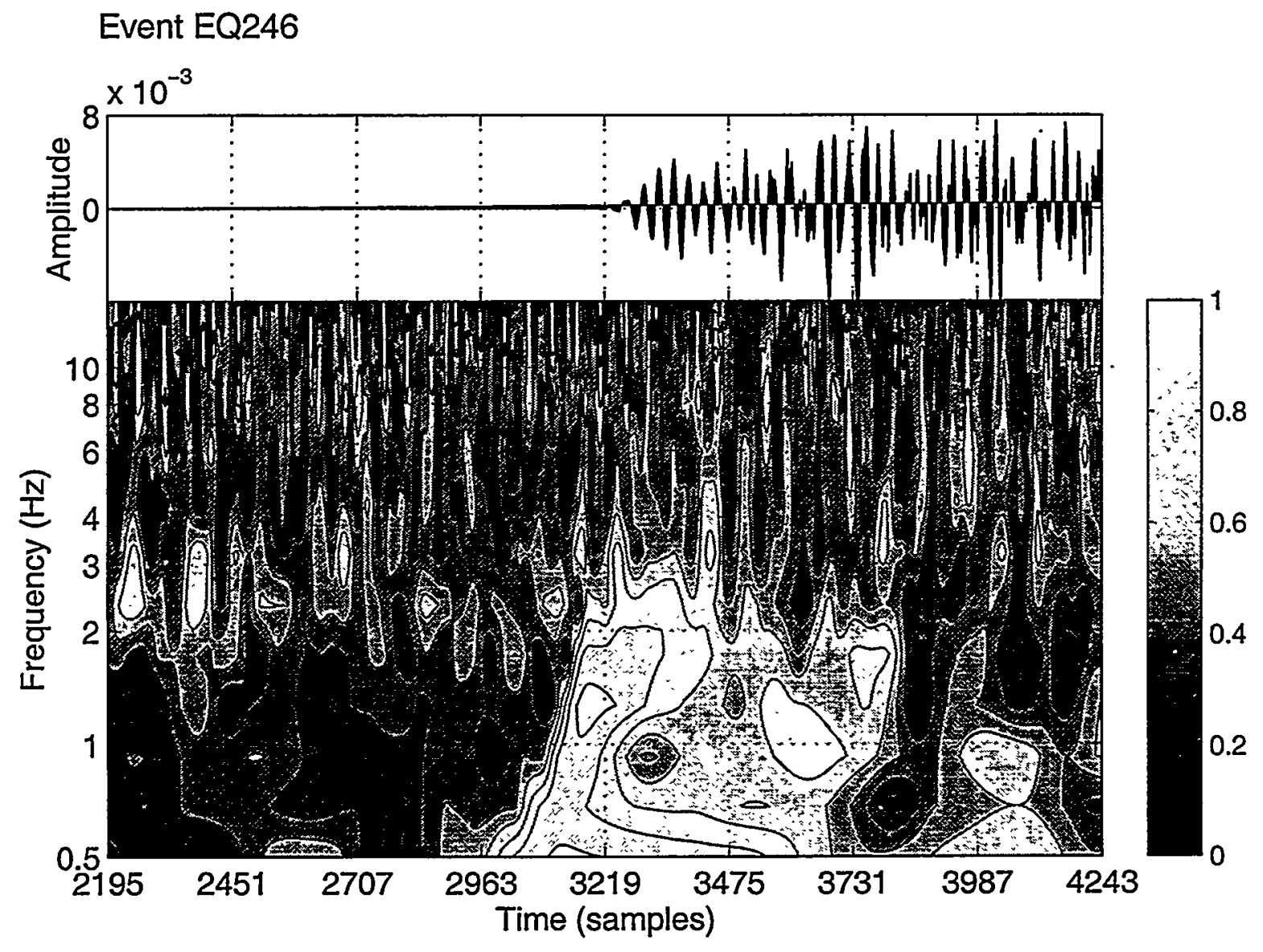

Figure 3. 


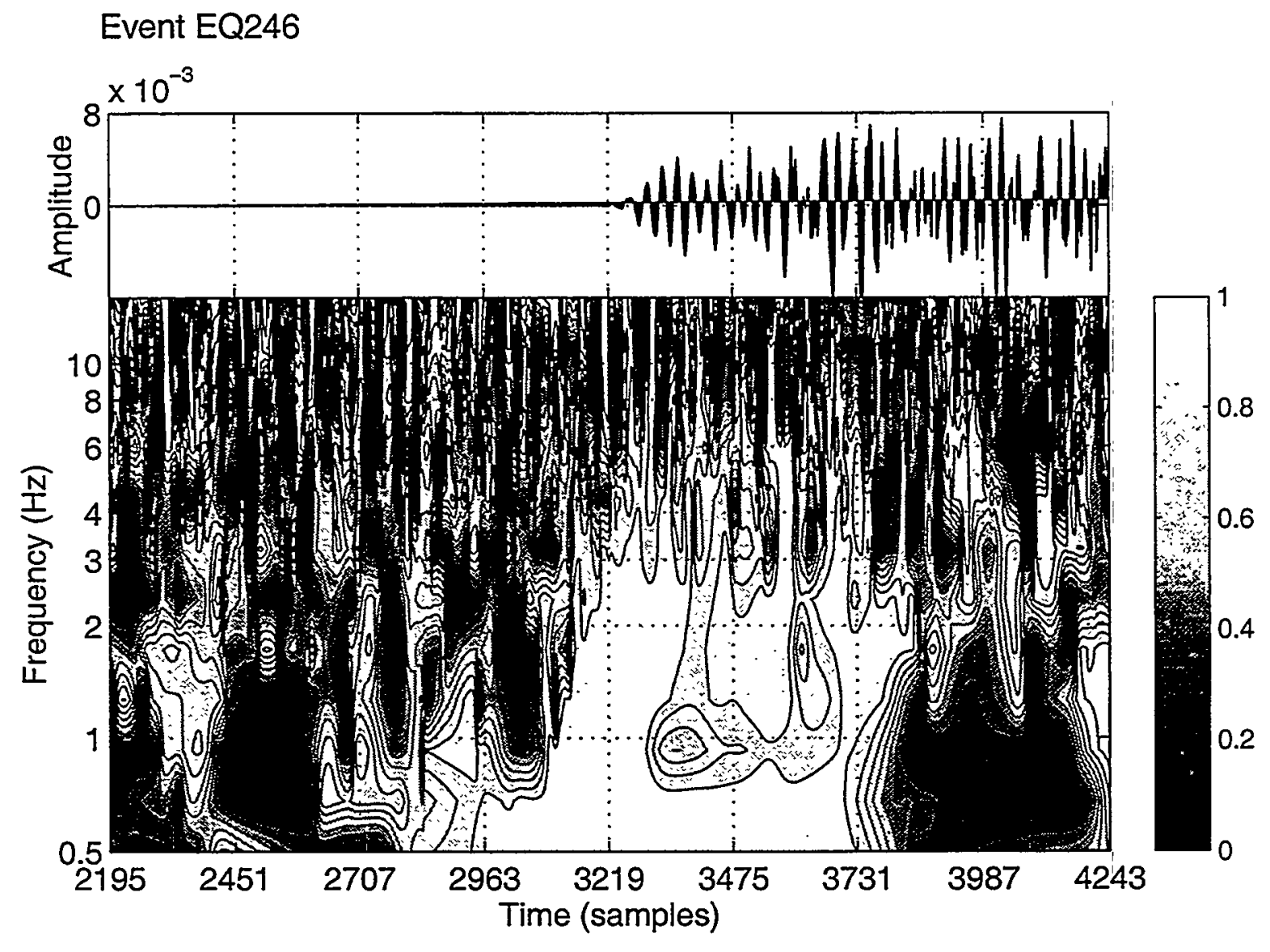

Figure 4. 


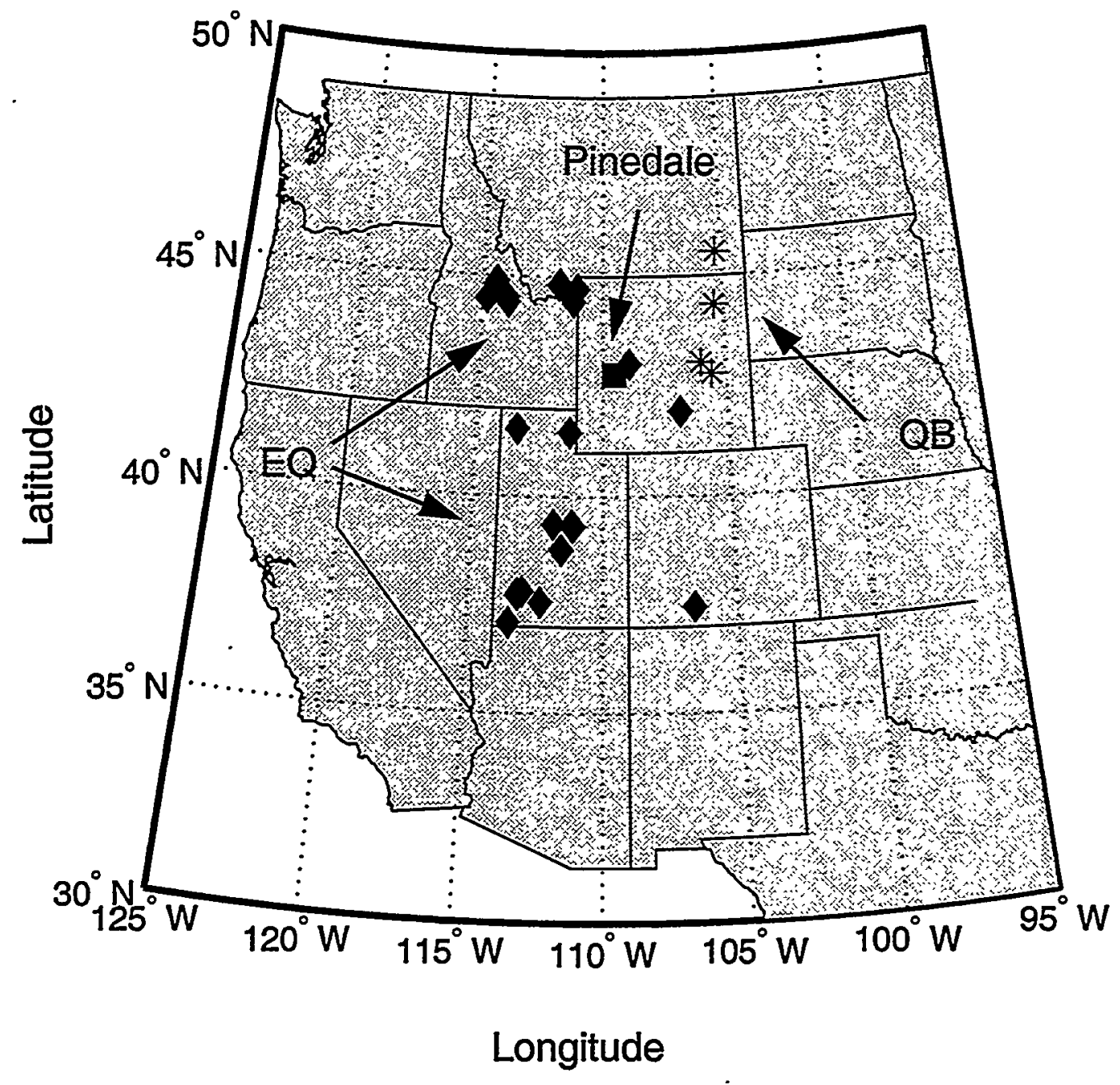

Figure 5. 
a) Signal trace and polarization spectrogram for event QB288

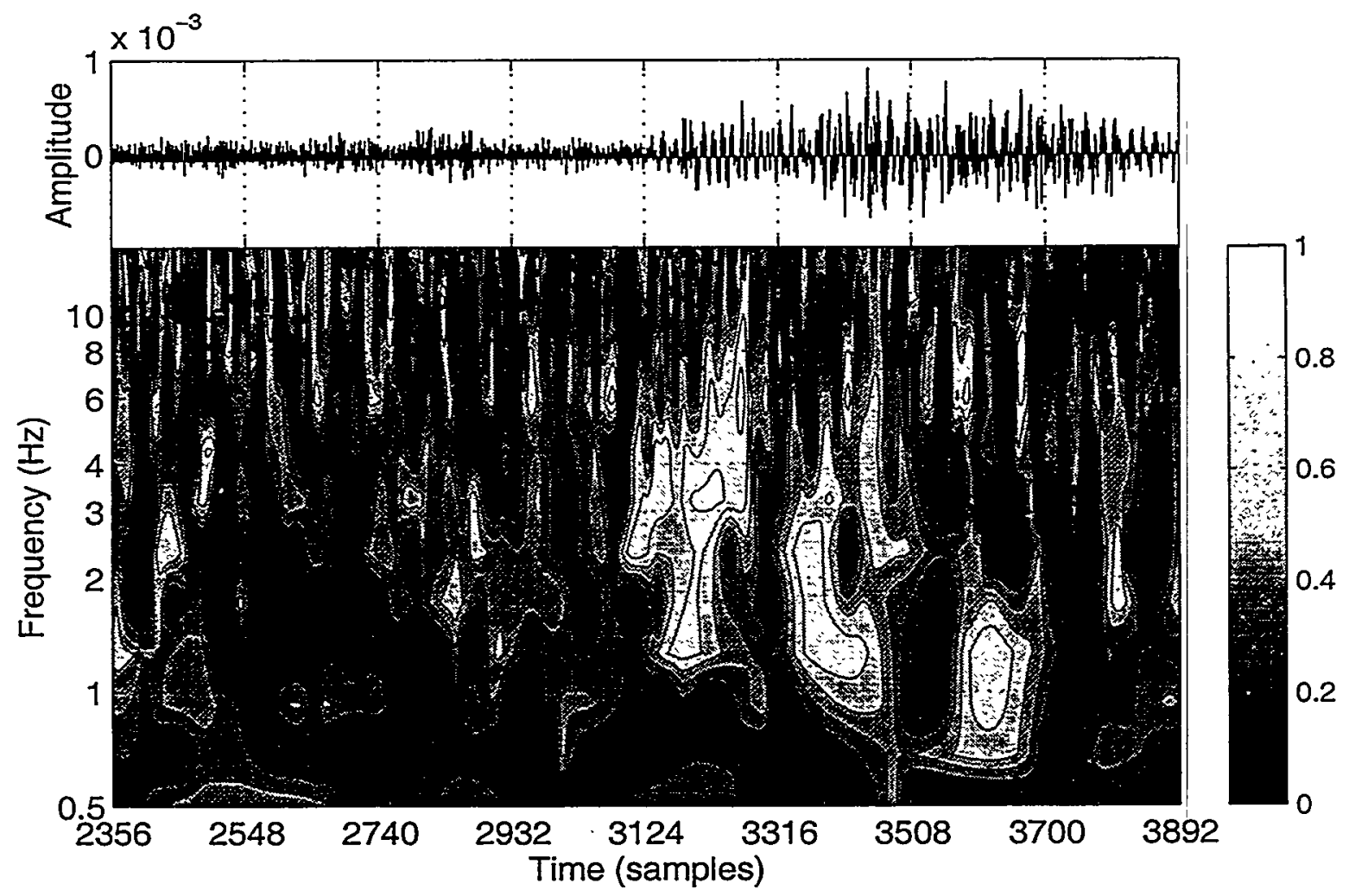

b) Bearing errors for event QB288

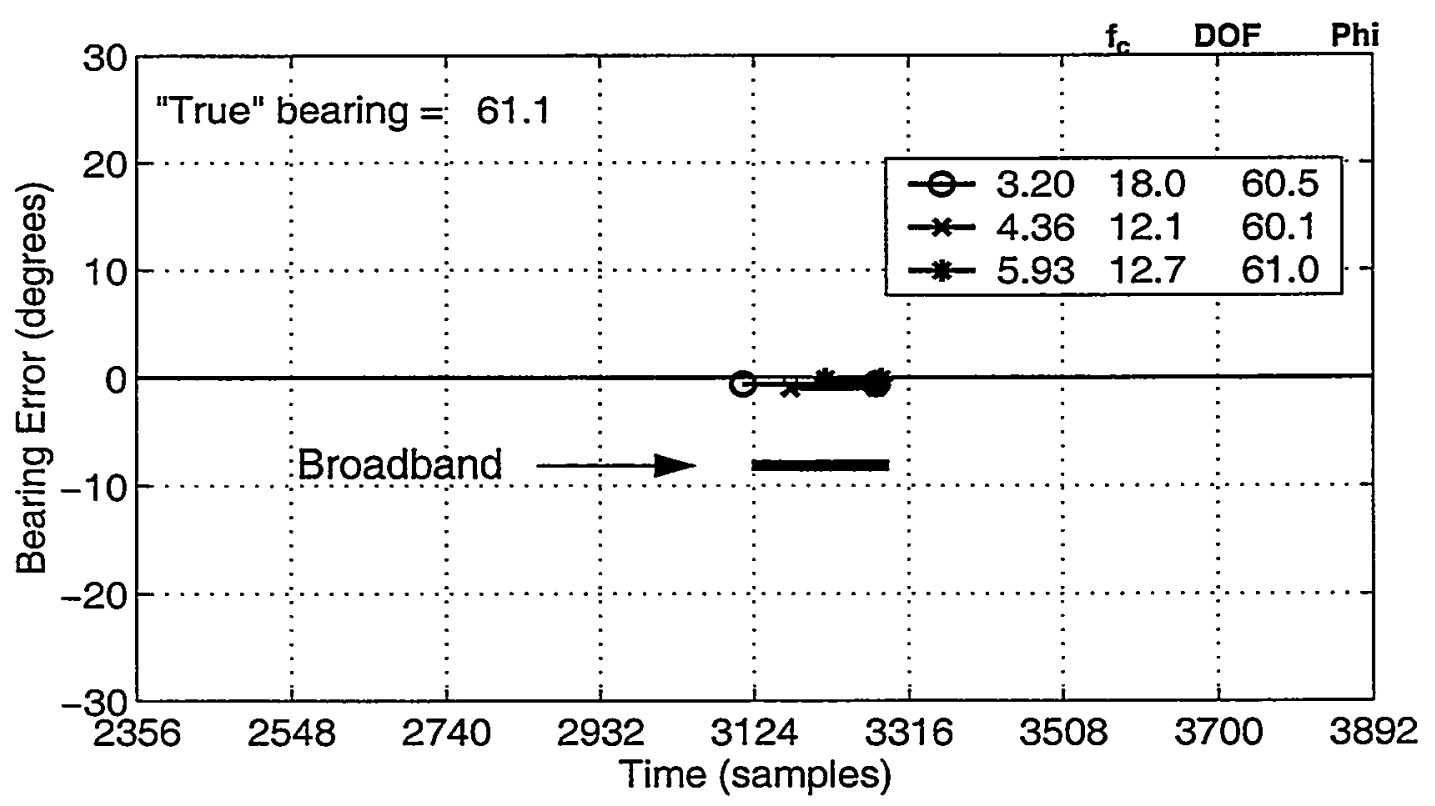

Figure 6. 
a) Signal trace and polarization spectrogram for event EQ246

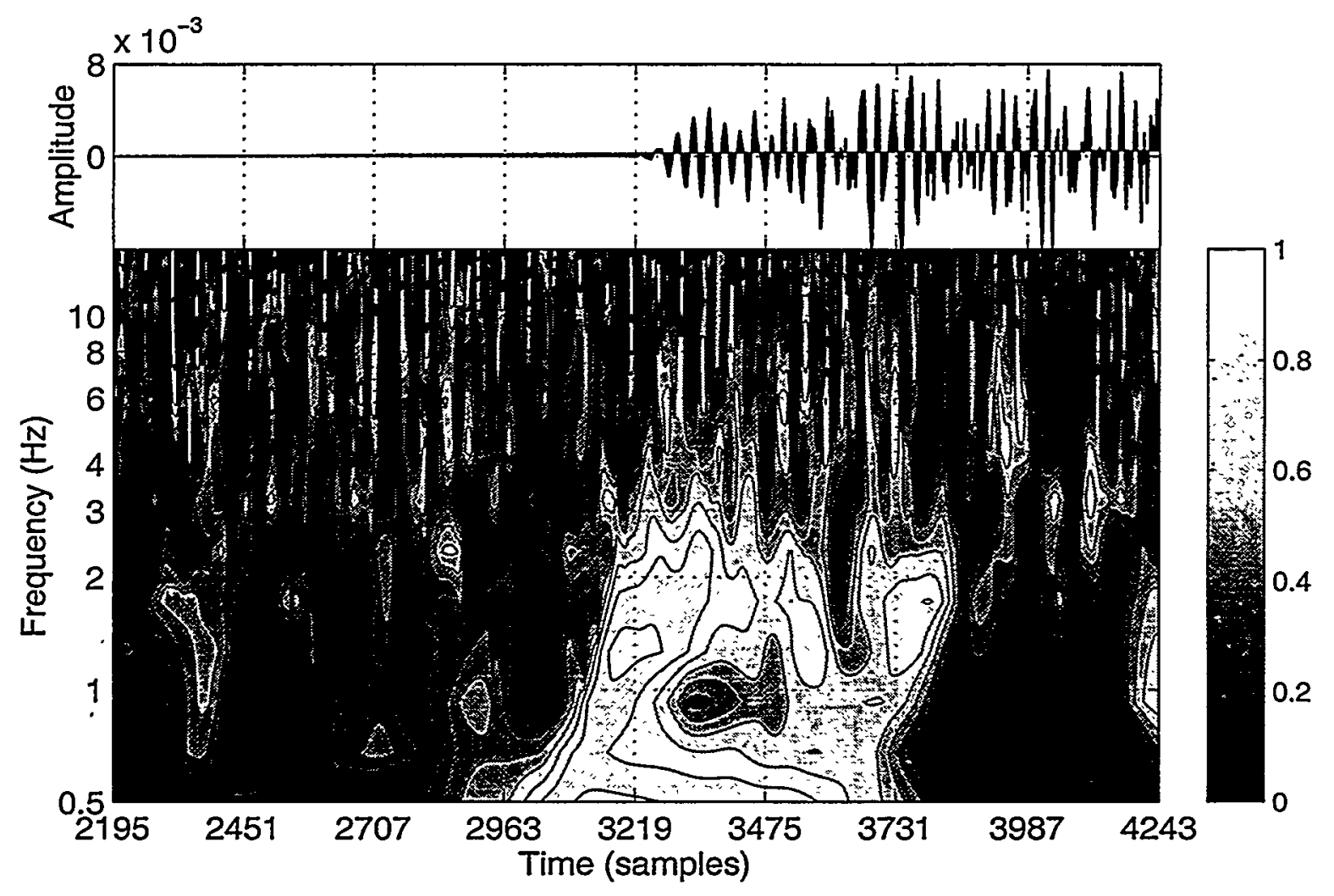

b) Bearing errors for event EQ246

$f_{c} \quad$ DOF Phi

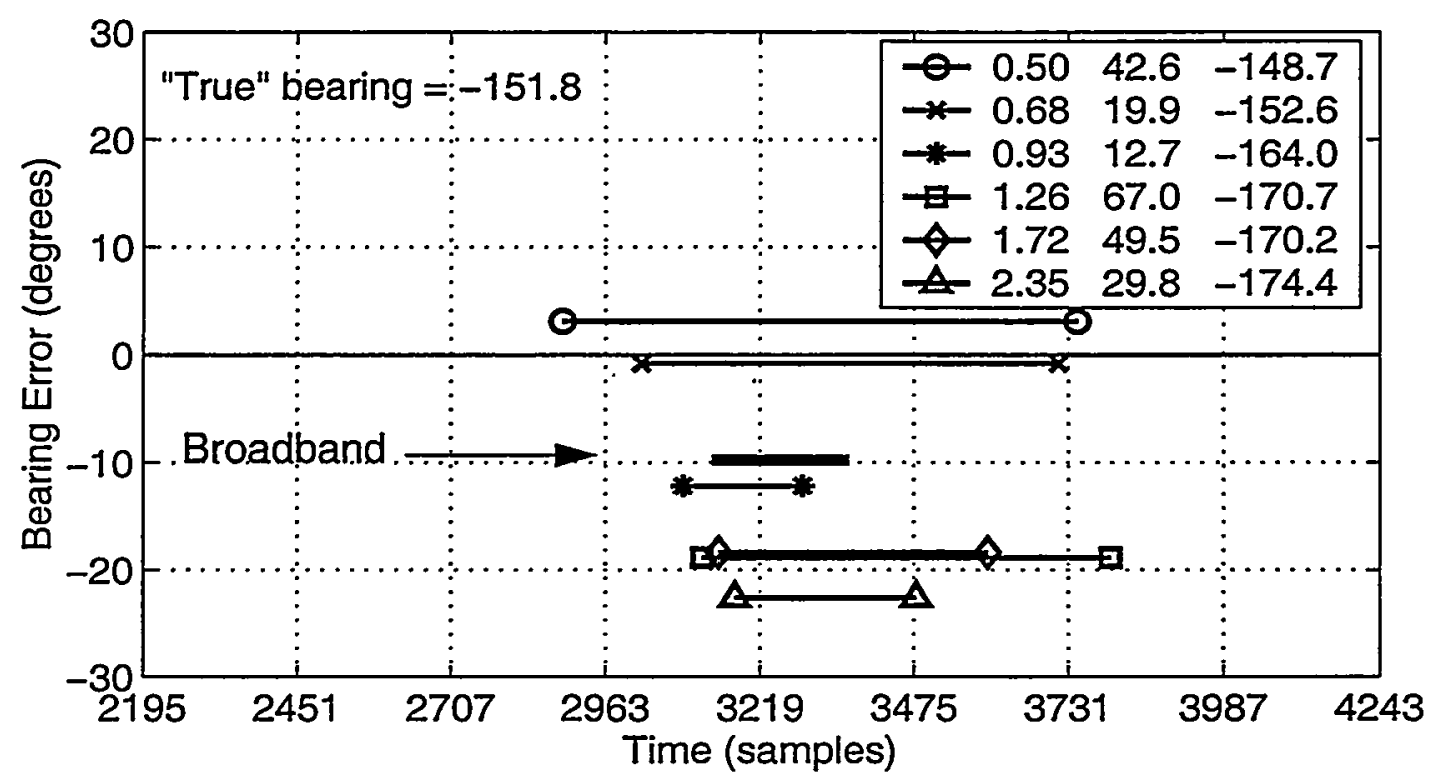

Figure 7. 
a) Signal trace and polarization spectrogram for event EQ031

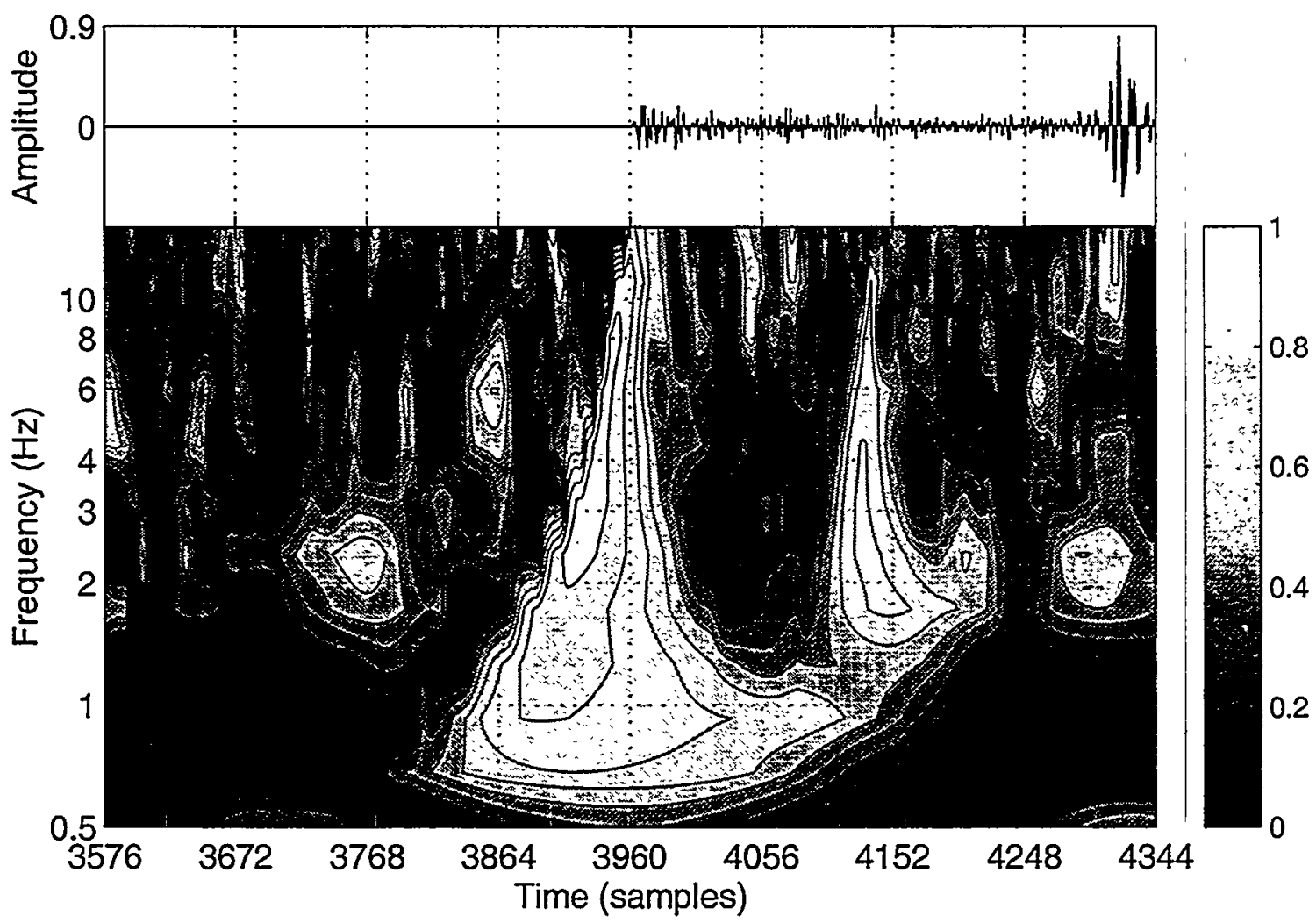

b) Bearing errors for event EQ031

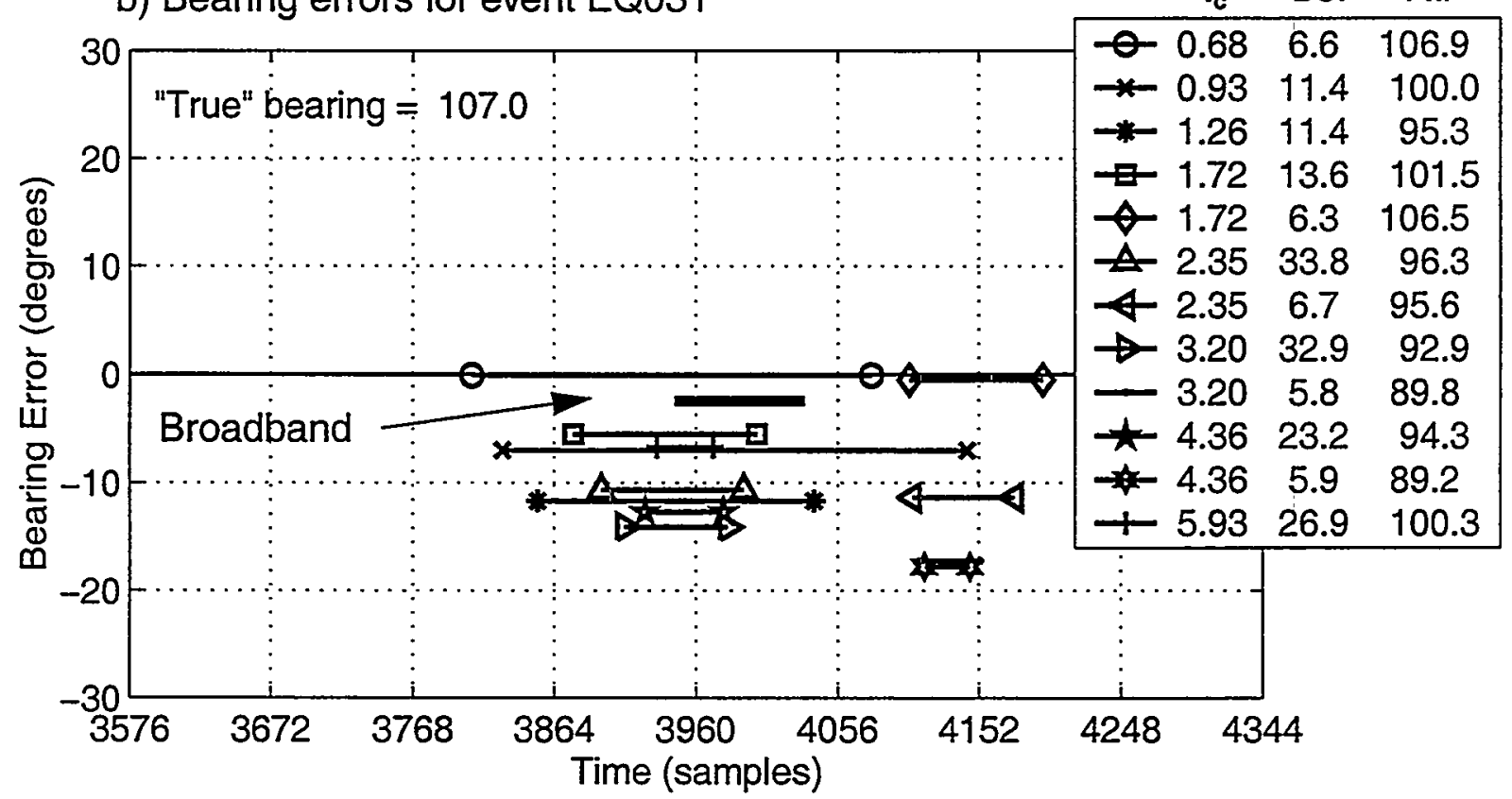

Figure 8. 
a) Signal trace and bearing errors for event EQ133

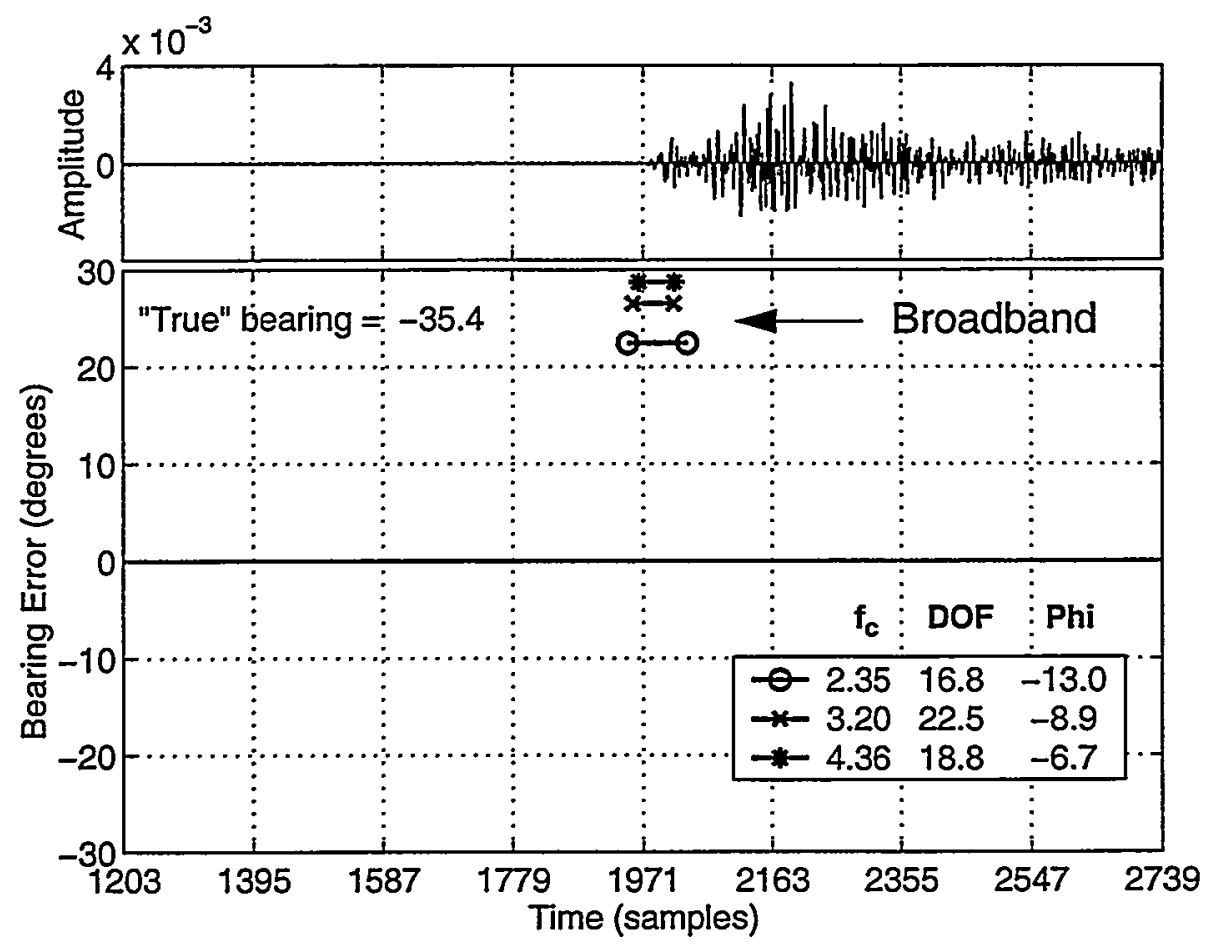

b) Signal trace and bearing errors for event EQ134

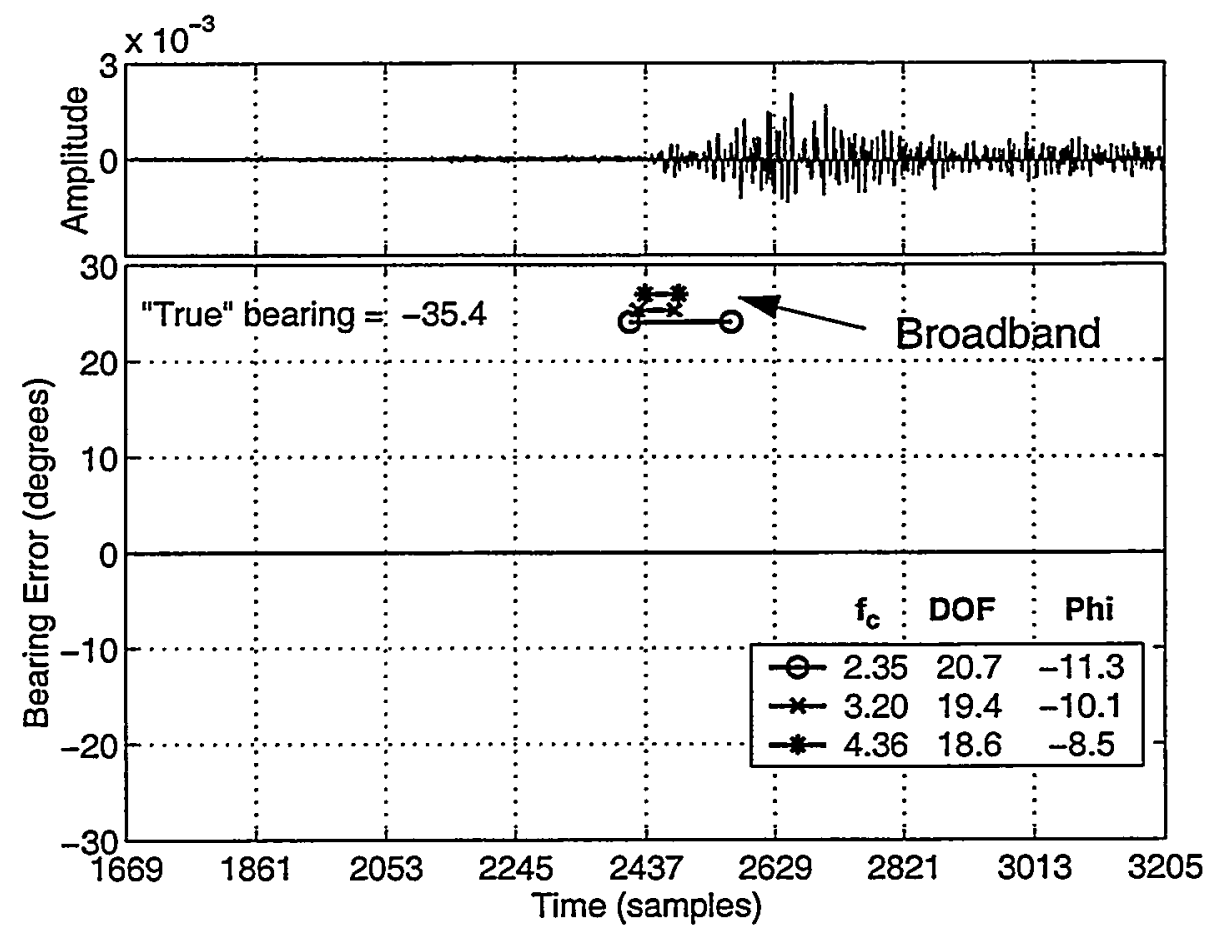

Figure 9. 
a) Signal trace and polarization spectrogram for event QB151

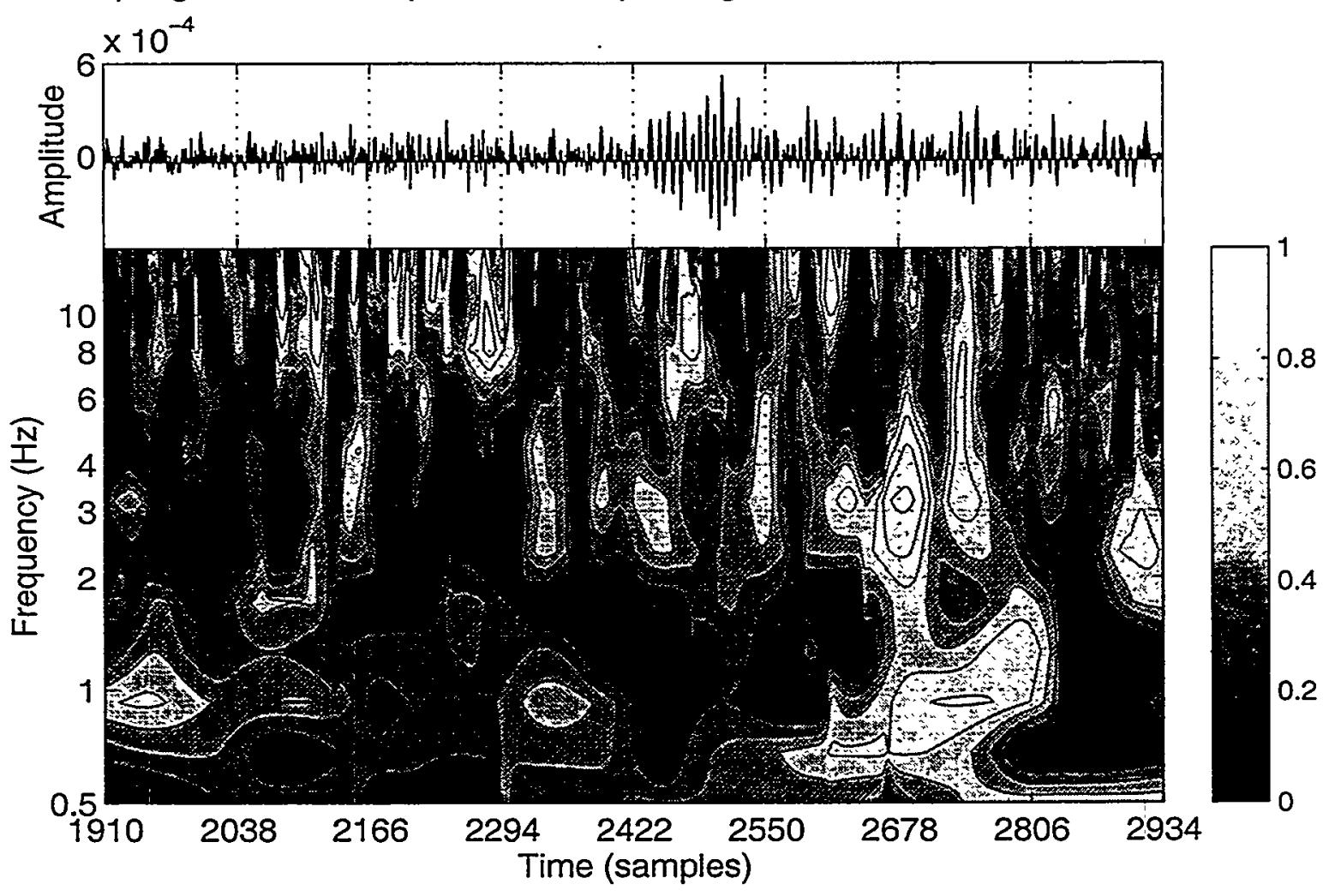

b) Bearing errors for event QB151

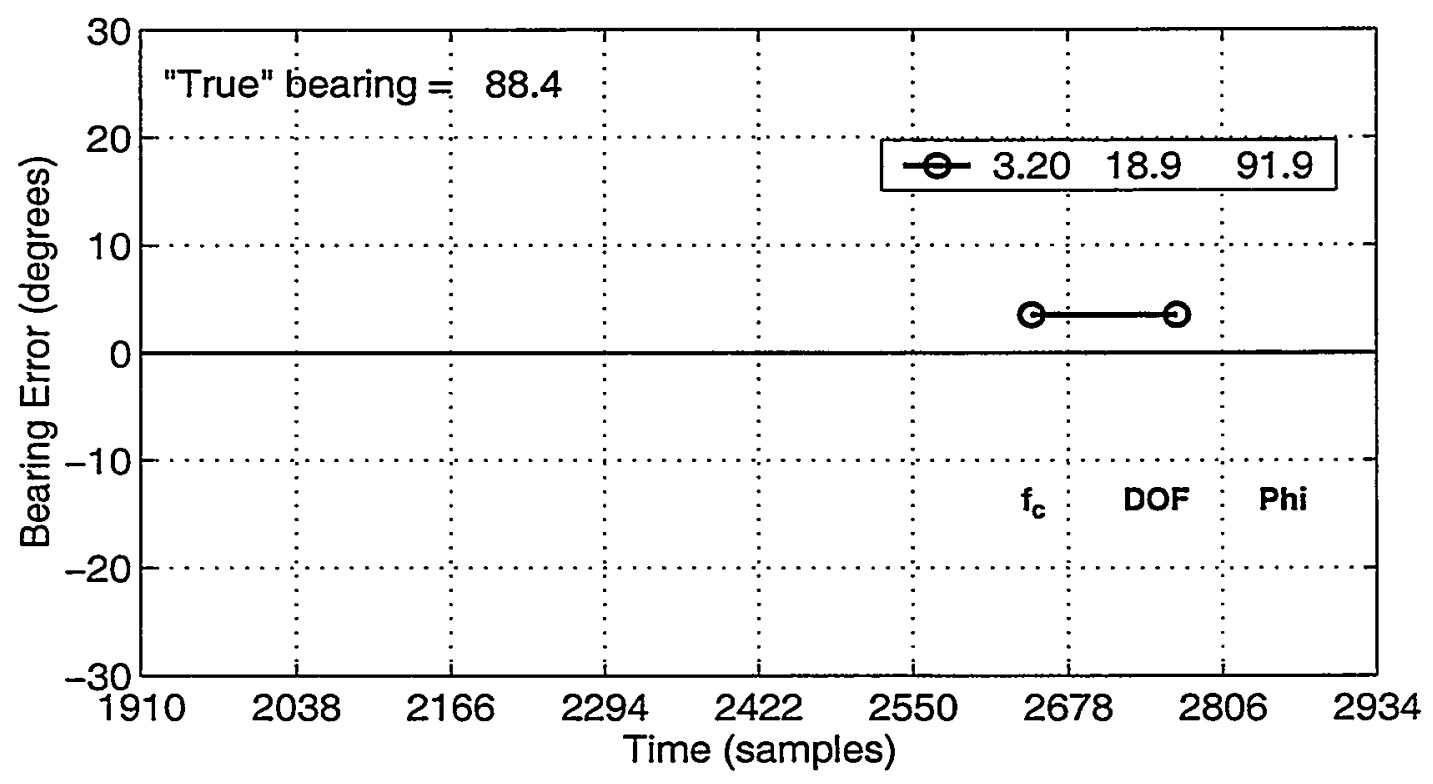

Figure 10. 


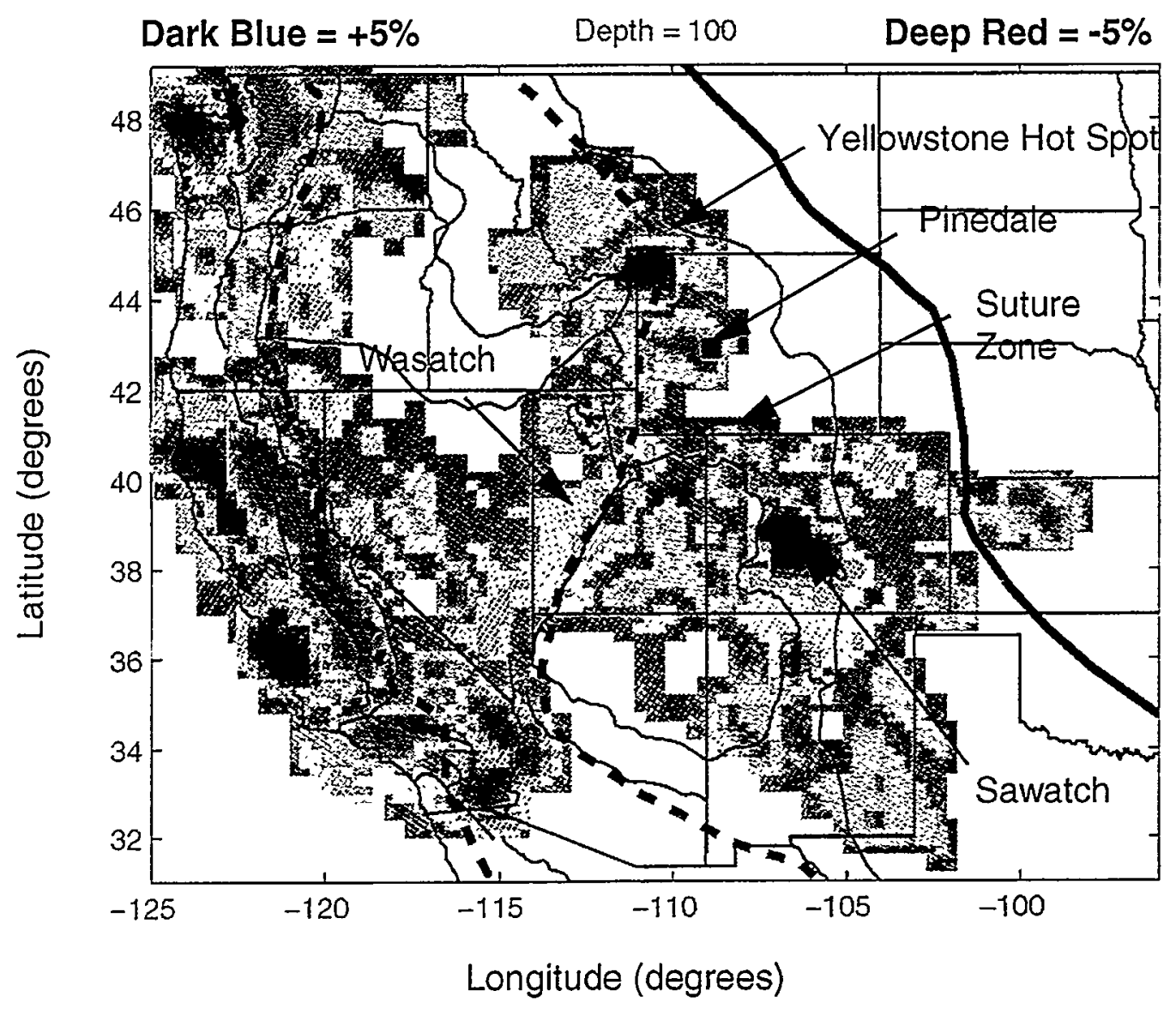

Figure 11. 
- 


\section{Distribution}

\section{External Distribution:}

US Department of Energy

Office of Research and Development

Attn: L. Casey

Forrestal Bldg. NN-20

1000 Independence Ave. SW

Washington, DC 20585

Los Alamos National Laboratory

Attn: W. Brunish, MS-F659

P.O. Box 1663

Los Alamos, NM 87545

Los Alamos National Laboratory

Attn: Steve Taylor, MS-C335

P.O. Box 1663

Los Alamos, NM 87545

Los Alamos National Laboratory

Attn: Aaron Velasco, MS-C335

P.O. Box 1663

Los Alamos, NM 87545

Lawrence Livermore National Laboratory

Attn: J. Zucca, MS L-205

P.O. Box 808

Livermore, CA 94550

Lawrence Livermore National Laboratory

Attn: C. Shultz, MS L-205

P.O. Box 808

Livermore, CA 94550

Lawrence Livermore National Laboratory

Attn: D. Harris, MS L-205

P.O. Box 808

Livermore, CA 94550
R. Warner, MS-KS-12

Pacific Northwest National Laboratories

P. O. Box 999

Richland, WA 99352

D. Hagedorn, MS-KS-12

Pacific Northwest National Laboratories

P. O. Box 999

Richland, WA 99352

K. Anderson, MS-KS-12

Pacific Northwest National Laboratories

P. O. Box 999

Richland, WA 99352

D. Anderson, MS-KS-12

Pacific Northwest National Laboratories

P. O. Box 999

Richland, WA 99352

\section{Internal Distribution:}

5704 D. A. Reynolds (MS-0980)

5704 D. P. Gallegos (MS-0980)

5736 P. B. Herrington (MS-0655)

5736 J. P. Claassen (MS-0655) (25 copies)

5736 J. M. Harris(MS-0655)

5736 E.P. Chael(MS-0655)

6116 C. J. Young (MS-0750

6533 D. B. Carr (MS-1138)

6533 P. L. Vaughan (MS-1138)

6533 R. G. Keyser (MS-1138)

4916 Technical Library (MS-0899) (2 copies)

8940-2 Central Technical Files (MS-9018)

04912 Review \& Approval Desk (MS-0612)

For DOE/OSTI 